\title{
OPEN Extracts from Pulsatilla patens target cancer-related signaling pathways in HeLa cells
}

\author{
Grażyna Łaska ${ }^{1}$, Magdalena Maciejewska-Turska², Elwira Sieniawska ${ }^{2 凶}$, Łukasz Świątek ${ }^{3}$, \\ David S. Pasco ${ }^{4}$ \& Premalatha Balachandran ${ }^{4}$
}

The purpose of this study was to determine if a methanolic extract of the Pulsatilla patens (L.) Mill. can inhibit the progression of cancer through the modulation of cancer-related metabolic signaling pathways. We analyzed a panel of 13 inducible luciferase reporter gene vectors which expression is driven by enhancer elements that bind to specific transcription factors for the evaluation of the activity of cancer signaling pathways. The root extract of $P$. patens exhibited strong inhibition of several signaling pathways in HeLa cells, a cervical cancer cell line, and was found to be the most potent in inhibiting the activation of Stat3, Smad, AP-1, NF-KB, MYC, Ets, Wnt and Hdghog, at a concentration of $40 \mu \mathrm{g} / \mathrm{mL}$. The methanolic extracts of $P$. patens enhanced apoptotic death, deregulated cellular proliferation, differentiation, and progression towards the neoplastic phenotype by altering key signaling molecules required for cell cycle progression. This is the first study to report the influence of Pulsatilla species on cancer signaling pathways. Further, our detailed phytochemical analysis of the methanolic extracts of the $P$. patens allowed to deduce that compounds, which strongly suppressed the growth and proliferation of HeLa cancer cells were mainly triterpenoid saponins accompanied by phenolic acids.

Cervical cancer is the third most common cancer as well as one of the leading causes of cancer-related death of women worldwide ${ }^{1}$. It causes about $13 \%$ of cancer cases in women. Cervical cancer epidemiology studies show that it is a serious oncological problem in developing countries, where $85 \%$ of the 500,000 cases are diagnosed diseases in the Word ${ }^{2}$. According to statistics in the world, the highest risk of disease is observed in Africa and South America, and in Europe, it is Romania, Bulgaria, and Serbia. The lowest incidence and mortality from cervical cancer occur in Australia, New Zealand, the United States, and Canada. About $60 \%$ of cases of this cancer occur in women aged $45-64^{2}$.

The first human cell line was obtained by George Gey in 1951 from a biopsy of cervical cancer, and was later named HeLa after the patient, Henrietta Lacks. Currently, these cells are one of the most frequently used cell lines in scientific research ${ }^{3}$. HeLa cells are aneuploid and divide rapidly ${ }^{4}$. These cells have a special character, resulting from altered chromosome number and structural disorder, compared to normal cells, which especially applies to chromosome $11,5,19, \mathrm{X}^{3,5}$. Studies of the HeLa genome confirmed the expression of genes responsible for the metabolic pathways connected with DNA repair and the cell cycle. Research by other authors has shown that chromothripsis occurs in $2-3 \%$ of all cancers ${ }^{6}$. It is believed, that chromothripsis and loss of heterozygosity, LOH on chromosome 11 contributed to the development of HeLa cervical cancer ${ }^{7}$. The use of the HeLa cell line allowed for the development of inactivated polio vaccine (IPV) by J. Salk in $1952^{8}$, description of human immunodeficiency virus (HIV) infection mechanism by R. Axel and E. Robey in $1986^{9}$, and the role of the human papillomavirus (HPV) in cancer transformation by H. Hansen in $1984^{10}$. Cervical dysplasia and cancer transformation may be the result of infection with the human papillomavirus (HPV). Hausen in 1982 proved that chronic HPV infection plays the role of a promoter of carcinogenesis of this tumor ${ }^{11}$, but other risk factors include early sexual intercourse, a large number of sexual partners, numerous pregnancies and deliveries at an early age, hormonal contraception, smoking tobacco and low economic status ${ }^{11}$. Currently, more than 200 types of HPV viruses have been detected. Of these, at least 85 types have been well known ${ }^{12}$. Due to their oncogenic potential, HPV viruses were divided into 3 groups: high, low and possibly low risk. Among the high-risk HPV

${ }^{1}$ Department of Agri-Food Engineering and Environmental Management, Bialystok University of Technology, 15-351 Bialystok, Poland. '2Department of Pharmacognosy, Medical University of Lublin, 20-093 Lublin, Poland. ${ }^{3}$ Department of Virology With SARS Laboratory, Medical University of Lublin, 20-093 Lublin, Poland. ${ }^{4}$ National Center for Natural Products Research, School of Pharmacy, University of Mississippi, University, Oxford, MS 38677, USA. ${ }^{\boxplus}$ email: esieniawska@pharmacognosy.org 
types, types 16 and 18 account for approximately $80 \%$ of all cervical cancer cases ${ }^{12}$. The oncogenic potential of some types of HPV results from the action of the E6 and E7 oncoproteins with transforming properties in the host cells. They disrupt tumor suppressor pathways and are essential for the proliferation of cervical cancer cells. Both oncoproteins are present in $99 \%$ of these cells ${ }^{13}$.

Natural products from Pulsatilla species have been used for centuries in traditional Chinese and Korean medicine for the treatment of many diseases and ailments. They are known as herbal drugs used for homeopathic treatment of neuralgia, insomnia, bronchitis, coughs, asthma, stress, anxiety, tension, skin eruptions, rheumatism, headaches, earache, eye ailments, hyperactivity, bacterial skin infections and malaria ${ }^{14}$. The Pulsatilla genus is composed of 38 species distributed in the northern hemisphere. Pulsatilla patens (L.) Mill. belongs to the Buttercup family (Ranunculaceae) commonly known as American Pasqueflower (synonym Anemone patens L.) but its chemical composition has not been extensively studied. P. patens is a lowland species of Boreo-meridionalcontinental distribution and it is native to Europe, Russia, Mongolia, China, Canada and United States ${ }^{15}$.

A complex network of signaling pathways is responsible for cellular proliferation, behavior and death in multicellular organisms. Research for the discovery of drugs targeting cancer has gained tremendous importance in the last few decades. Recently, transcription factors (Stat3, Smad, AP-1, NF- $\kappa$ B, E2F, MYC, Ets, Notch, FoxO, Wnt, Hdghog, miR-21, k-Ras) have attracted a fair share of attention as a novel drug target for cancer treatment. These are key transcription factors that regulate cytological processes, including differentiation, cell death, proliferation, oncogenic transformation and apoptosis ${ }^{16}$. Of the several hundred cancer-associated genes and driver gene mutations identified thus far, the vast majority belong to approximately $13+$ different signaltransduction pathways. In our research, we have collected a panel of 13 inducible luciferase reporter gene vectors (Stat3, Smad, AP-1, NF-kB, E2F, MYC, Ets, Notch, FoxO, Wnt, Hdghog, miR-21, k-Ras), where expression is driven by enhancer elements that bind to specific transcription factors ${ }^{17}$. These vectors can be used to assess the activity of cancer-related signaling pathways. These are signaling nodes for multiple oncogenic pathways, which transduces intracellular and extracellular signals to the nucleus and control the expression of genes responsible for multiple physiological processes such as cell growth, proliferation, differentiation, positioning, migration, metabolism and apoptosis ${ }^{16}$.

Our study aimed to assess the effect of methanolic extracts from roots and leaves of the Pulsatilla patens (L.) Mill. in inhibiting the progression of human cancer cells by selected cancer-related signaling pathways. Different vectors as transcription factors in the signal transduction pathways were studied (Stat3, Smad, AP-1, NF- $\mathrm{B}, \mathrm{E} 2 \mathrm{~F}$, MYC, Ets, Notch, FoxO, Wnt, Hdghog, miR-21, k-Ras, pTK - control), in the presence of various inducers (IL6 , TGF- $\beta$, PMA, wnt-3a), when cells were exposed to extracts for 4-6 h. Besides, we also performed a detailed phytochemical analysis of this species which allowed for identification of triterpenoid saponins and phenolic acids responsible for the activity.

\section{Results}

Results of cytotoxicity studies. The methanolic extracts from roots of $P$. patens showed cytotoxicity to all the cell lines included in the assay (Table 1). The $\mathrm{IC}_{50}$ concentrations for cytotoxicity for $P$. patens were in the range of $32-58 \mu \mathrm{g} / \mathrm{mL}$ for each cell line indicating a general cytotoxic activity throughout the panel of cancer and non-cancer cells.

The cancer-related signaling pathways in HeLa cells. The activity of cancer-related signaling pathways was assessed using a panel of luciferase reporter gene vectors, in which luciferase expression is driven by the binding of transcription factors to multiple copies of synthetic enhancers within each vector. The methanolic extracts from the roots of $P$. patens were more powerful than the compounds from leaf extract of this species inhibiting the activation of 11 pathways analysed, with the exception only two, FoxO and miR-21. The active compounds of methanolic extracts from the roots of $P$. patens were found to be the most potent in inhibiting the activation of Stat3, Smad, AP-1, NF- $\kappa$ B, MYC, Ets, Wnt and Hdghog signaling (Fig. 1). E2F and Notch signaling were inhibited stronger by compounds of root extract than leaf extract, but similarly inhibited as by an active antitumor compound used in the research, resveratrol analog. Similarly, the activation of the apoptotic mediators pathways FoxO, miR-21 and k-Ras were not observed with either compounds from the methanolic extracts of $P$. patens (Supplementary Table S1).

The methanolic extracts from the roots of $P$. patens were stronger in potency than active antitumor compound resveratrol analog in each of their concentrations for inhibition of signaling mediated by Stat3, Smad, AP-1, NF- $\kappa B, E 2 F, M Y C$, Ets, Wnt and Hdghog, but were similar in potency as resveratrol analog for the inhibition of signaling mediated by Notch and k-Ras. However, the resveratrol analog was more effective than active compounds of $P$. patens for FoxO and miR-21. It was found that methanolic extracts from the roots of $P$. patens did not inhibit luciferase expression driven by the minimal thymidine kinase promoter (pTK-control), indicating it as not significant for general cytotoxicity or luciferase enzyme inhibition.

Since active compounds of methanolic extract from the root of $P$. patens were more forceful than from leaves of this species with respect to inhibiting the activation of pathways, it was determined which concentration of this extract was also a more effective modulator of cancer-related signaling pathways. Active compounds of methanolic extracts from roots of $P$. patens with a concentration of $40 \mu \mathrm{g} / \mathrm{mL}$ were stronger than these compounds from other concentrations $(30 \mu \mathrm{g} / \mathrm{mL}$ and $15 \mu \mathrm{g} / \mathrm{mL}$ ) (Supplementary Table S1). Active compounds from roots of $P$. patens with a concentration of $40 \mu \mathrm{g} / \mathrm{mL}$ were more potent inhibiting the activation of 12 signaling pathways used, with the exception of only two, FoxO and miR-21. The active compounds from the roots of $P$. patens with a concentration of $40 \mu \mathrm{g} / \mathrm{mL}$ were found to be the most effective in inhibiting the activation of 11 signaling 


\begin{tabular}{|c|c|c|c|c|c|c|c|}
\hline Sample name & $\mathbf{K B}^{\mathbf{b}}$ & BT-549c & SK-OV-3 ${ }^{\mathrm{d}}$ & SK-MEL ${ }^{\mathrm{e}}$ & $\mathrm{HeLa}^{\mathrm{f}}$ & LLC-PK-1 ${ }^{\mathrm{g}}$ & Vero $^{h}$ \\
\hline \multicolumn{8}{|c|}{ Cytotoxic activity $\left(I C_{50} \mu g / m L\right)^{a}$} \\
\hline $\begin{array}{l}\text { Root extract } \\
\text { P. patens }\end{array}$ & 38 & 32 & 32 & 34 & 58 & 36 & 32 \\
\hline Doxorubicin* & 1.7 & 2.2 & 2.3 & 1.7 & 3.9 & 1.6 & $>5$ \\
\hline
\end{tabular}

Table 1. Cytotoxicity activities of $P$. patens methanolic extracts from roots towards a panel of mammalian cell lines. ${ }^{\mathrm{a}} \mathrm{IC}_{50} \mu \mathrm{g} / \mathrm{mL}$ is the concentration that affords $50 \%$ inhibition of cell growth. ${ }^{\text {b-h }}$ Human cell lines of epidermal carcinoma, breast carcinoma, ovarian carcinoma, skin melanoma, cervical carcinoma, kidney epithelial cells, and kidney fibroblast respectively, ${ }^{*}$ positive control drug.

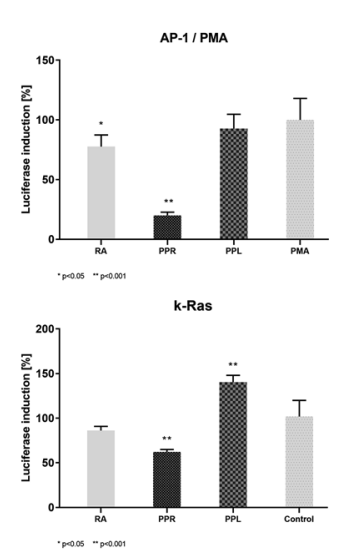

PTK

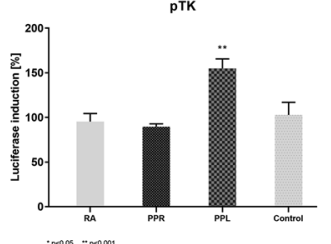

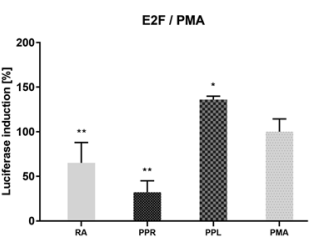

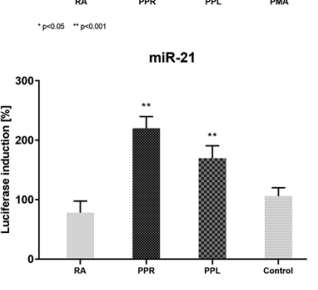

Smad / TGF- $\beta$

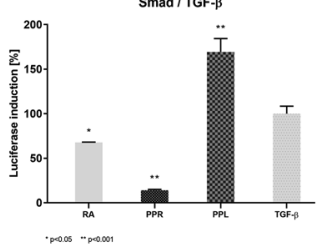

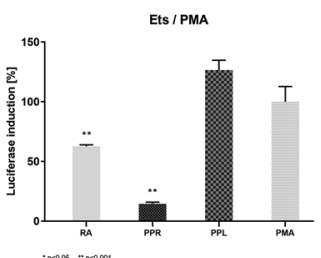

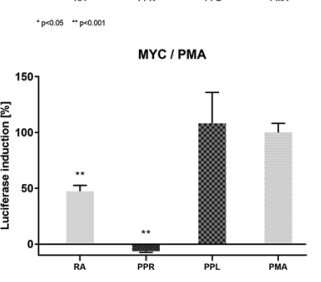

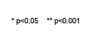

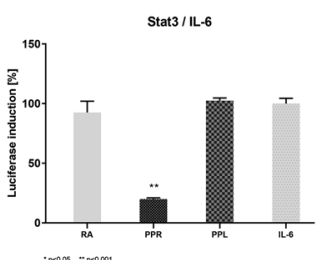

proces " posos
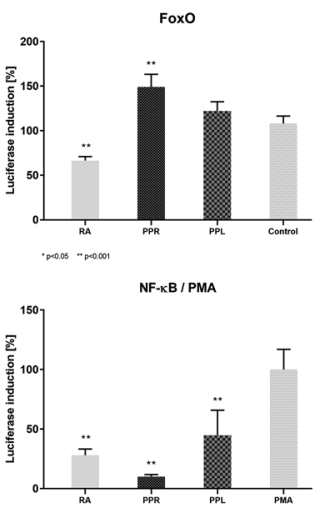

worcs pesoso

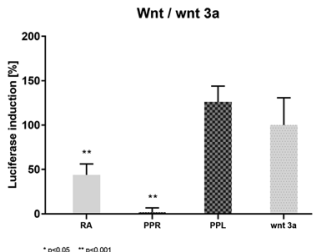

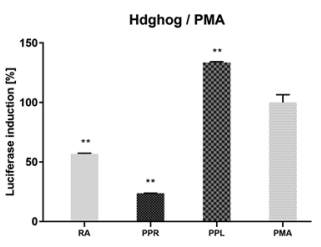

Notch / PMA

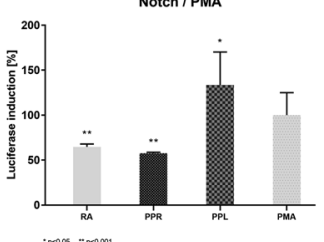

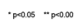

RA - Resveratrol analog $(6 \mu \mathrm{M})$ PPR - Pulsatilla patens root extract $(40 \mathrm{\mu g} / \mathrm{mL})$ $\mathrm{PPL}-$ Pulsatilla patens leaves extract $(40 \mathrm{\mu g} / \mathrm{mL})$ PMA - Phorbol 12-myristate 13-acetate TGF-B - Transforming growth factor beta IL-6 - Interleukin 6 wnt $3 a-$ Wnt family member $3 A$

Figure 1. The inhibition of activations of Stat3, Smad, MYC, Ets, E2F, Ap-1, NF- $\kappa$ B, Wnt, Hdghog and pTK-control by methanolic extract of Pulsatilla patens and reference/control compound-resveratrol analog. IL-6, TGF- $\beta$, PMA, wnt 3a - Inducer (promoter) of cancer processes. Resveratrol analog - the analog 3,5,4'-trihydroxy-trans-stilbene, trans-resveratrol or $(E)$-resveratrol-is a stilbenoid, a type of natural phenol, and a phytoalexin with anticancer activity. The obtained data were statistically analyzed using GraphPad Prism (two-way ANOVA, Dunnett's multiple comparisons test).

pathways used, such as Stat3, Smad, AP-1, NF- $\mathrm{B}$, MYC, Ets, Wnt, Hdghog, E2F, Notch and k-Ras (Fig. 2). None of the extracts with the concentrations of $40 \mu \mathrm{g} / \mathrm{mL}, 30 \mu \mathrm{g} / \mathrm{mL}$ and $15 \mu \mathrm{g} / \mathrm{mL}$ inhibited the apoptotic mediators FoxO, miR-2 1 and inhibited the minimal thymidine kinase promoter (pTK control) in the luciferase expression, indicating a weak general cytotoxicity.

This is the first report of active compounds of methanolic extracts from roots of $P$. patens from a natural source influencing the cancer-related signaling pathways in HeLa cells, and also the first report of different 13 signaling pathways as transcription factors in cancer processes from the genus Pulsatilla patens. Obtained data suggests that the action of $P$. patens methanolic extract is based on the induction of apoptosis and on the inhibition of mitosis by disturbing the HeLa cell cycle (Fig. 3). The small-molecule kinase inhibitors, which, through selectivity and specificity, inhibit the activation of specific proteins, also act on the receptors responsible for oncogenesis. Based on the studied cancer-related signaling pathways in HeLa cells we propose that the methanolic extracts of $P$. patens enhance apoptotic death, deregulated cellular proliferation, differentiation, and progression towards the neoplastic phenotype by altering key signaling molecules required for cell cycle progression. Thereby, this mechanism prevents excessive, harmful proliferation of the HeLa cells and inhibits them.

The active compounds of methanolic extracts of $P$. patens. The results showed that $P$. patens is a rich source of polyphenolic constituents, mainly saponins and hydroxycinnamic acids. Interestingly, $P$. patens showed a different profile of triterpenoid saponins in comparison to other species from the Ranunculaceae family.

The calibration curves of reference compounds were linear in the range of $0.02-1 \mathrm{mg} / \mathrm{mL}$ and $0.002-0.5 \mathrm{mg} /$ $\mathrm{mL}$, for Pulsatilla saponin $\mathrm{D}$ and ferulic acid, respectively. The linearity was very good with the correlation coefficient $\left(\mathrm{R}^{2}\right)$ values higher than 0.999 , as shown in Table 2 . The results obtained in the intraday and interday precision measurements of both reference standards were below $1 \%$, indicating the high stability of the method. 


\section{Dose-response activity of $P$. patens roots methanolic extract} on signaling pathways

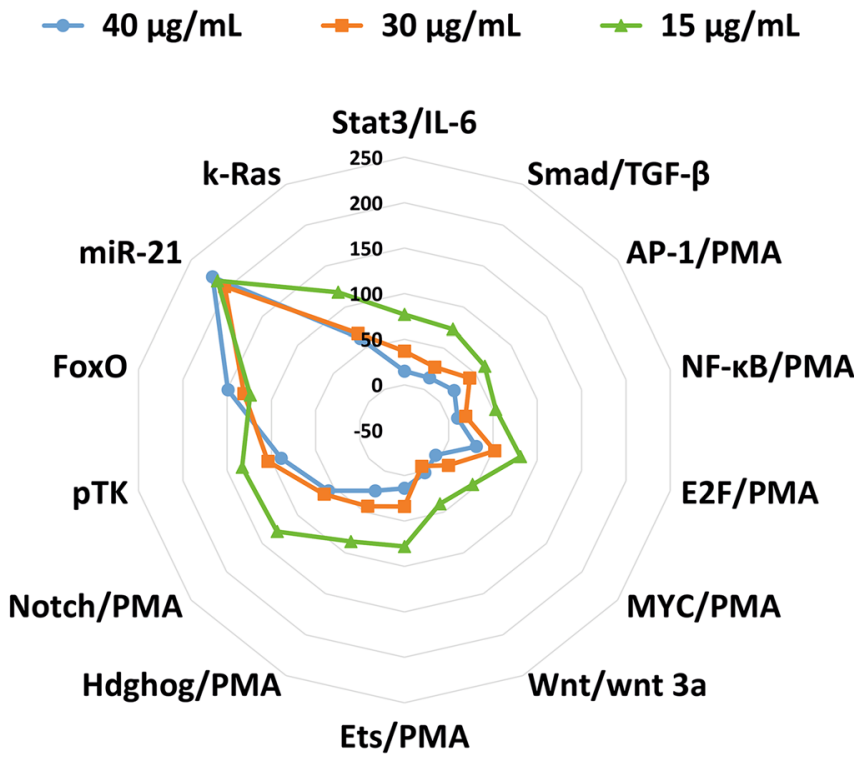

Figure 2. The active compounds from the roots of $P$. patens against cancer-related signaling pathways in HeLa cells with different concentrations of $40 \mu \mathrm{g} / \mathrm{mL}, 30 \mu \mathrm{g} / \mathrm{mL}$ and $15 \mu \mathrm{g} / \mathrm{mL}$.

The recovery was in the range from $87.5 \%$ (RSD 0.89\%) for Pulsatilla saponin D to $97.04 \%$ (RSD $0.49 \%$ ) for ferulic acid.

The analyzed compounds vary remarkably with the part of the plant. The total content of phenolic acids was higher in leaves while roots were found to be an abundant source of triterpenoid saponins, representing the dominant class of constituents. The content of saponins was in the range from 0.11 to $2.14 \mathrm{mg} / \mathrm{g} \mathrm{dw}$ in roots and from 0.14 to $0.32 \mathrm{mg} / \mathrm{g} \mathrm{dw}$ in the leaves (Table 3; Supplementary Fig. S1). Triterpenoid saponins and phenolic acids present in $P$. patens extracts were characterized and tentatively identified for the first time. Their MS data were listed in Table 4. Most of the triterpenoid saponins found in the studied P. patens extracts shared common features. The characteristic solvent adduct ions $\left[\mathrm{M}+\mathrm{HCOO}^{-}\right.$or deprotonated ions $[\mathrm{M}-\mathrm{H}]^{-}$were usually observed. The composition of sugar residues was deducted based on the fragmentation pathway observed in MS/MS spectra. The peaks with high abundance intensities were formed from the typical for negative ionization mode, simultaneous loss of $470 \mathrm{Da}$, which was attributed to the cleavage of the ester bond and the elimination of the entire sugar chain, assigned as Glc-Glc-Rha, from C-28 position ${ }^{18}$. Whereas the successive losses of sugar moieties, such as $162 \mathrm{Da}$ (-Glc), $146 \mathrm{Da}$ (-Rha) or $132 \mathrm{Da}$ (-Ara) correspond to the sequence and composition of sugar fragments linked to C- $3^{16}$. According to the literature data, the majority of saponins with a molecular weight higher than $1000 \mathrm{Da}$ found in Pulsatilla taxa were determined to be bidesmosidic saponins substituted at both C-3 and C-28 positions with oligosaccharides ${ }^{19}$.

P. patens contained two sapogenin skeletons, determined as lupane (Lup-type) and oleane-type (Olean-type) (Fig. 4), which produced characteristic fragment ions at $m / z=471^{19}$. These fragment ions may be related to both 23-hydroxybetulinic acid (anemosapogenin) and hederagenin structure what may explain the existence of isomers in the analyzed samples. According to Jin et al. ${ }^{18}$ these two types of aglycones can be distinguished by their different retention times resulting from smaller Clog P-value of Lup-type, and consequent earlier elution of Olean-type saponins on the reversed-phase column. Additionally, several peaks shared a similar fragmentation pathway but were assigned to other than hederagenin, olenane-type oligoglycosides, which primarily yielded an abundant ion at $m / z 487$ suggesting the presence of bayogenin structure, reported previously by ${ }^{20}$ in another species $P$. patnes var. multifida.

The detailed spectrometric data revealed that peaks 22, 25 and 28 shared the same basic skeleton of bayogenin identified based on the characteristic fragment ion at $\mathrm{m} / z 487$, which further generated a product ion at $\mathrm{m} / z 471$ as a result of the neutral elimination of $\mathrm{H}_{2} \mathrm{O}^{19}$. The difference between analyzed compounds originated from residues at an $\alpha$-sugar chain. The observation of precursor ions at $m / z 943,781$ and 619 for compounds $\mathbf{2 2}, \mathbf{2 5}$ and 28, respectively resulted from the elimination of the entire $\beta$-sugar chain from C-28 position. For compound 22, based on the product ions with values of 781, 619 and 487, we deduced that two Glc and one Ara molecule were linked to C-3 position. Therefore, the chemical structure of peak number $\mathbf{2 2}$ was tentatively established as 3-O-glucopyranosyl-glucopyranosyl-arabinopyranosyl-bayogenin 28-O-rhamnopyranosyl(1-4)-glucopyrano syl(1-6)-glucopyranosyl ester, while compounds 25 and 28 as 3-O-glucopyranosyl- arabinopyranosyl-bayogenin 28-O-rhamnopyranosyl(1-4)-glucopyranosyl(1-6)-glucopyranosyl ester and 3-O-arabinopyranosyl-bayogenin 28-O-rhamnopyranosyl(1-4)-glucopyranosyl(1-6)-glucopyranosyl ester, respectively. 


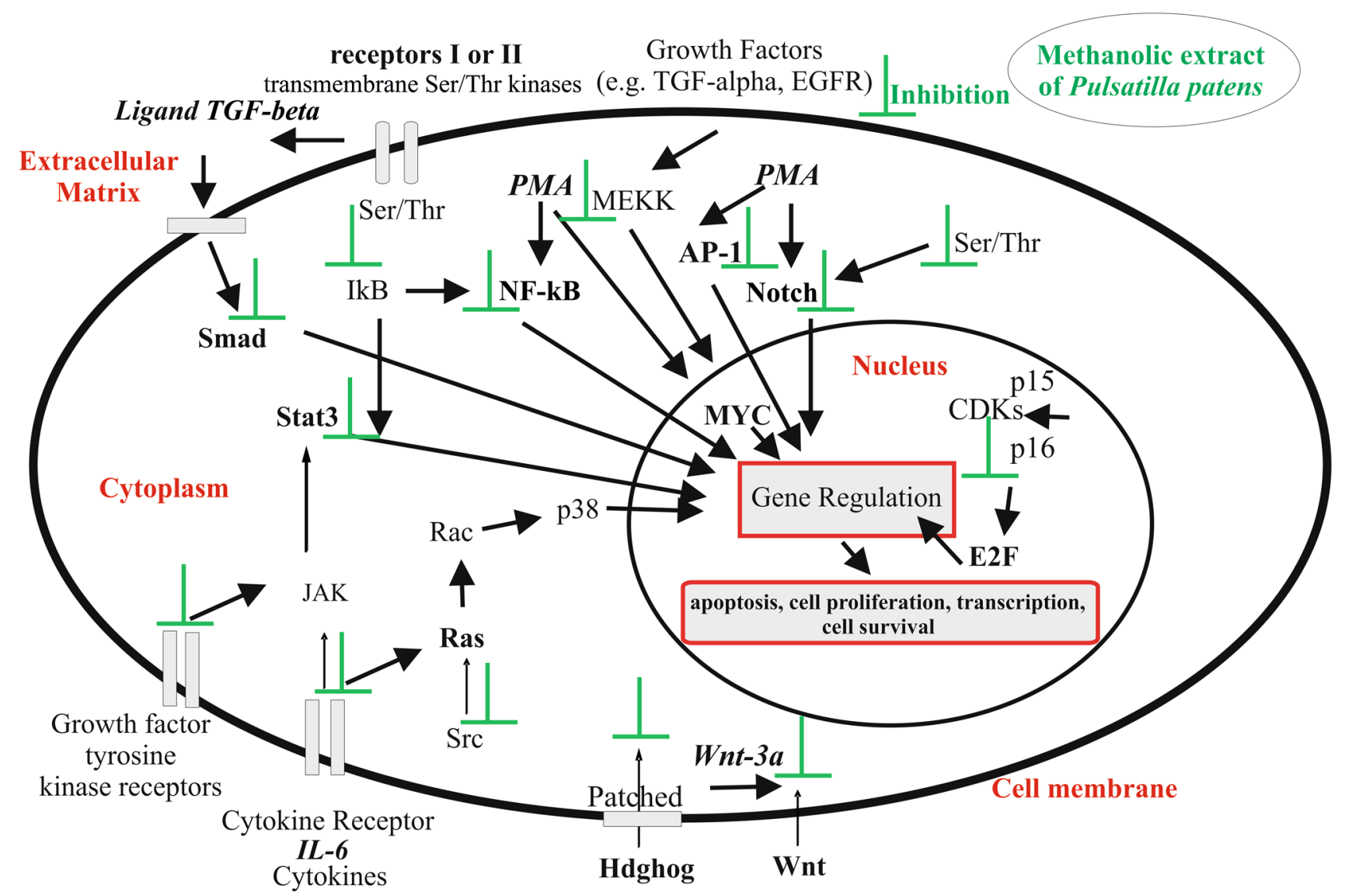

Figure 3. Cancer-related signaling pathways in HeLa cells inhibited by $P$. patens methanolic extract and the mechanism of extract $P$. patens action in the cancer cell. The methanolic extracts of $P$. patens enhance apoptotic death, deregulated cellular proliferation, differentiation, and progression towards the neoplastic phenotype by altering key signaling molecules required for cell cycle progression. Stat3, Smad, AP-1, NF- $\kappa B, E 2 F, M Y C$, Notch, Wnt, Hdghog, Ras Transcription factors, Cytokines IL-6, Ligand TGF-beta Inducers, PMA Protein kinase activator, phorbol 12-myristate-13-acetate, Wnt-3a protein, Ser/Thr Serine/threonine kinases, EGFR Epidermal growth factor receptor, MEKK Mitogen-activated protein (MAP) kinase, IkB Inhibitor of kB, JAK Janus kinase, Src Protooncogene tyrosine-protein kinase, Rac Subfamily of the Rho family of GTPases, Ras Ras family kinase, p38 Mitogen-activated protein kinases, p15, p16 The cyclin-dependent kinase inhibitors, CDKs Cyclins, cyclindependent kinases, Patched Protein patched homolog.

\begin{tabular}{|c|c|c|c|c|c|c|c|c|}
\hline $\begin{array}{l}\text { Reference } \\
\text { substance }\end{array}$ & $\begin{array}{l}\text { Regression } \\
\text { equation }\end{array}$ & $R^{2}$ & $\begin{array}{l}\text { Linearity range } \\
(\mathrm{mg} / \mathrm{mL})\end{array}$ & LOD (mg/mL) & LOQ (mg/mL) & $\begin{array}{l}\text { Concentration } \\
\text { range }(\mathrm{mg} / \mathrm{mL})\end{array}$ & $\begin{array}{l}\text { Intraday precision } \\
(n=3)(\%)\end{array}$ & $\begin{array}{l}\text { Interday precision } \\
(n=3)(\%)\end{array}$ \\
\hline \multirow{2}{*}{ Pulsatilla saponin D } & \multirow{2}{*}{$y=857.12 x-99.356$} & \multirow{2}{*}{0.9995} & \multirow{2}{*}{$0.02-1$} & \multirow{2}{*}{0.0461} & \multirow{2}{*}{0.1396} & 0.05 & 0.45 & 0.57 \\
\hline & & & & & & 0.25 & 0.26 & 0.11 \\
\hline \multirow{2}{*}{ Ferulic acid } & \multirow{2}{*}{$y=8715.9 x+241.52$} & \multirow{2}{*}{0.9998} & \multirow{2}{*}{$0.002-5$} & \multirow{2}{*}{0.0107} & \multirow{2}{*}{0.0326} & 0.005 & 0.76 & 0.55 \\
\hline & & & & & & 0.01 & 1.07 & 0.57 \\
\hline
\end{tabular}

Table 2. Method validation parameters for reference substances; linearity, precision, LOD, LOQ and quantification.

Peak 24 produced an abundant precursor ion $[\mathrm{M}-\mathrm{H}]^{-}$at $m / z 1397$. Further fragmentation of this molecule generated product ion at $m / z 927$ derived from neutral losses of Glc-Glc-Rha sugar chain from the C-28 position. The MS/MS spectra revealed three products ions at $m / z$ valued 765,603 and 471 , due to the sequential loss of Glc, Glc, Ara from C-3 position thus peak 24 was presumed to be 3-O-glucopyranosyl-glucopyranosyl-arabinopyranosyl hederagenin 28-O-rhamnopyranosyl-(1-4)-glucopyranosyl-(1-6)-glucopyranosyl ester.

Compounds 27 and 29 showed the same formula of $\mathrm{C}_{59} \mathrm{H}_{96} \mathrm{O}_{27}$ and yielded identical precursor ions [M- $\left.\mathrm{H}\right]^{-}$at $\mathrm{m} / \mathrm{z}$ 1235. Compared with peak 24 the molecular weight of these two compounds was $162 \mathrm{Da}$ less, suggesting the lack of one glucose in the $\beta$-sugar chain. Both $\mathbf{2 7}$ and $\mathbf{3 0}$ exhibited similar fragmentation patterns. The characteristic fragment ion with $\mathrm{m} / z$ value of 765 indicated the elimination of the entire Glc-Glc-Rha from C-28. The detection of ions at $m / z 603$ and 471 at compound 27 was attributed to the sequential loss of Glc 


\begin{tabular}{|c|c|c|}
\hline \multirow[b]{2}{*}{ COMPOUND } & \multicolumn{2}{|c|}{$\begin{array}{l}\text { Content (mg/g } \\
\text { DW) }\end{array}$} \\
\hline & Leaves & Roots \\
\hline \multicolumn{3}{|l|}{ Saponins } \\
\hline 3-O-GlcGlcAra, 28-O-RhaGlcGlc bayogenin (22) & - & 0.14 \\
\hline 3-O-GlcGlcAra, 28-O-RhaGlcGlc hederagenin (24) & - & 1.10 \\
\hline 3-O-GlcAra, 28-O-RhaGlcGlc bayogenin (25) & - & 0.59 \\
\hline 3-O-GlcAra, 28-O-RhaGlcGlc 23-hydroxybetulinic acid (27) & - & 2.14 \\
\hline 3-O-Ara, 28-O-RhaGlcGlc bayogenin $(\mathbf{2 8})$ & 0.23 & 0.47 \\
\hline 3-O-GlcAra, 28-O-RhaGlcGlc hederagenin (29) & 0.32 & 1.84 \\
\hline 3-O-Ara, 28-O-RhaGlcGlc 23-hydroxybetulinic acid (30) & 0.15 & 0.97 \\
\hline 3-O-Ara-Rha, 28-O-RhaGlcGlc hederagenin/3-O-Ara-Rha, 28-O-RhaGlcGlc 23-hydroxybetulinic acid (32) & 0.17 & - \\
\hline 3-O-AraGlc 23-hydroxybetulinic acid (33) & - & 0.12 \\
\hline 28-O-RhaGlcGlc 23-hydroxybetulinic acid (36) & 0.16 & 0.12 \\
\hline 3-O-AraGlc 23-hydroxybetulinic acid (37) & - & 0.11 \\
\hline 3-O-Ara, 28-O-Glc hederagenin (41) & - & 0.12 \\
\hline 3-O-Ara, 28-O-Glc hederagenin (42) & 0.14 & 0.14 \\
\hline \multicolumn{3}{|l|}{ Hydroxycynnamic acid type compounds } \\
\hline Dicaffeic acid (5) & 0.03 & 0.01 \\
\hline Coumaric acid (6) & - & 0.01 \\
\hline Caftaric acid (7) & 0.08 & - \\
\hline Caffeic acid (8) & 0.02 & 0.02 \\
\hline Ferulic acid (20) & 0.32 & 0.07 \\
\hline Chicoric acid isomer 1 (23) & 0.65 & 0.30 \\
\hline Chicoric acid isomer $2(\mathbf{2 6})$ & 0.17 & 0.10 \\
\hline Coumaric acid derivative $(\mathbf{3 1})$ & 0.04 & - \\
\hline
\end{tabular}

Table 3. Content of saponins and hydroxycynnamic acid type compounds in P. patens extracts. Ara arabinose; Glc - glucose; Rha - rhamnose; DW - dry weight.

and Ara, respectively. The identical ions found in both analyzed compounds confirmed the existence of the same sugar chain at C-3. Saponin $\mathbf{2 7}$ and $\mathbf{2 9}$ were expected to be isomers with different aglycone structure belonging to lupane or hederegenin. The saponin 27 eluted earlier at $29.407 \mathrm{~min}$ was characterized as Lup-type and tentatively identified as Pulsatilloside $\mathrm{D}^{18,21}$, whereas saponin 29 eluted at 30.282 min as Leonloside D (=leiyemudanoside $)^{14,18}$.

The compound 30 characterized with an adduct ion $\left[\mathrm{M}+\mathrm{HCOO}^{-}\right.$at $\mathrm{m} / z 1119$, gave the precursor ion at $\mathrm{m} / z$ 1073 , which was $162 \mathrm{Da}$ less than that of peak 29, corresponding to the lack of one Glc group in the $\beta$-sugar chain of compound 30. Comparing with the fragmentation of compound 29 and appearance of similar product ions at $m / z 603$ we could deduce that C-28 residues correspond to Glc-Glc-Rha, whereas ions observed at $m / z 471$, resulted from a loss of one Ara moiety from C-3. Thus saponin 30 eluted at 31.265 min was identified as 3-Oarabinopyranosyl-23-hydroxybetulinic acid 28-O-rhamnopyranosyl(1-4)-glucopyranosyl(1-6)-glucopyranosyl ester. Consequently, its isomer 35 eluted later at $33.889 \mathrm{~min}$ was assigned as 3-O-arabinopyranosyl-hederagenin 28-O-rhamnopyranosyl(1-4)-glucopyranosyl(1-6)-glucopyranosyl ester.

Another pair of isomers 38 and 40, which produced identical precursor ions at $m / z 617$ have been found. Further fragmentation yielded ion at $m / z 471$ indicating the neutral elimination of Rha moiety from both saponins. In the MS/MS spectra of compounds 38 and $\mathbf{4 0}$ the characteristic ion at $m / z 247$, formed from the ring cleavage reaction has been observed. Unfortunately, in this case, data derived from negative-ion mode was insufficient to assume the location of Rha fragment neither in C-3 nor C-28 position. Based on the differences in elution behavior of analyzed compounds, saponin $\mathbf{3 8}$ was assigned as Lup whereas $\mathbf{4 0}$ as Olean-type and they have proposed to be 23-hydroxybetulinic acid $O$-rhamnopyranoside and hederagenin $O$-rhamnopyranoside, respectively ${ }^{18}$.

Interestingly, four isomeric compounds 33, 37, 41 and 42 showed the same molecular formula of $\mathrm{C}_{41} \mathrm{H}_{66}$ $\mathrm{O}_{13}$ and afforded an adduct ion $\left[\mathrm{M}+\mathrm{HCOO}^{-}\right.$at $\mathrm{m} / z$ 811, which fragmented in slightly different ways. For saponins 41 and 42 the most prominent precursor ion was observed at $m / z 765$ and further fragmentation of $[\mathrm{M}-\mathrm{H}]^{-}$formed product ions at $\mathrm{m} / z 603$ and 471 , which correspond to one Glc (-162 Da) in either C-3 or C-28 and one Ara (-132 Da) molecule in C-3 position. While for the second pair of isomers 33 and 37 an abundant product ion at $m / z 603$, correlating to neutral loss of Glu, was observed in MS/MS spectrum. The differences in fragmentation behavior of analyzed isomers and their fragment ions abundances might originate not only from aglycone structure but also from the variation in C-3 or C-28 composition. The MS data obtained from negative-ion mode was insufficient to clearly determine the localization of glucose moiety. The elution behavior of analyzed compounds and literature data, indicate the slightly more polar saponins $\mathbf{3 3}$ and $\mathbf{3 7}$ eluted at 33.510 and $35.472 \mathrm{~min}$, respectively to be Lup rather than Olean-type saponins, and tentatively identified as two isomers of $O$-arabinopyranosyl-glucopyranosyl 23-hydroxybetulinic acid ester, while $\mathbf{4 1}$ and $\mathbf{4 2}$ isomers were proposed 


\begin{tabular}{|c|c|c|c|c|c|c|c|c|c|}
\hline Comp. No & Tentative identification & Rt (min) & Molecular fromula & MW & {$[\mathrm{M}-\mathrm{H}]^{-}$} & Fragments $(\mathbf{m} / \mathbf{z})$ & Roots & Leaves & References \\
\hline 1. & Caffeic acid hexoside & 1.622 & C15H18O9 & 342.0947 & 341.0947 & $179.0559 ; 161.0539 ; 135.0455$ & + & + & 29,60 \\
\hline 2. & $\begin{array}{l}\text { Dihydroxybenzoic acid } \\
\text { hexoside }\end{array}$ & 6.303 & C13H16O9 & 316.0766 & 315.0766 & $153.0567 ; 109.0337$ & + & + & 27,31 \\
\hline 3. & Hydroxymelilotic acid & 8.988 & $\mathrm{C} 9 \mathrm{H} 10 \mathrm{O} 4$ & 182.0544 & 181.0544 & $163.0402 ; 135.0487$ & + & + & - \\
\hline 4. & Hydroxybenzoic acid isomer 1 & 9.656 & $\mathrm{C} 7 \mathrm{H} 6 \mathrm{O} 3$ & 138.0276 & 137.0276 & $119.0236 ; 108.0210 ; 93.0393$ & + & + & 27 \\
\hline 5. & Dicaffeic acid & 10.471 & C15H18O9 & 342.0947 & 341.0947 & $179.0361 ; 161.0223 ; 135.0455$ & + & + & 60 \\
\hline 6. & Coumaric acid & 13.002 & $\mathrm{C} 9 \mathrm{H} 8 \mathrm{O} 3$ & 164.0433 & 163.0428 & 149.0126; 135.0450; 119.0503 & + & - & 60 \\
\hline 7. & Caftaric acid & 13.07 & $\mathrm{C} 13 \mathrm{H} 12 \mathrm{O} 9$ & 312.0457 & 311.0457 & $\begin{array}{l}\text { 179.0332; 149.0079; 135.0406; } \\
112.9989\end{array}$ & - & + & 30 \\
\hline 8. & Caffeic acid & 16.745 & $\mathrm{C} 9 \mathrm{H} 8 \mathrm{O} 4$ & 180.0392 & 179.0392 & $135.0456 ; 107.05$ & + & + & 27,28 \\
\hline 9. & Anemonin & 17.621 & $\mathrm{C} 10 \mathrm{H} 8 \mathrm{O} 4$ & 192.0391 & 191.0391 & $147.0470 ; 101.0357$ & + & - & 61 \\
\hline 10. & Vanilic acid & 18.505 & $\mathrm{C} 8 \mathrm{H} 8 \mathrm{O} 4$ & 168.0385 & 167.0385 & $152.0090 ; 108.0228$ & + & + & 30 \\
\hline 11. & Ferulic acid deriv & 19.544 & & 226.0810 & 225.0810 & $193.0516 ; 135.0475$ & - & + & 27 \\
\hline 12. & Unknown & 19.549 & & 682.2496 & 681.2496 & $519.1772 ; 357.1296 ; 151.0426$ & + & - & - \\
\hline 13. & Ferulic acid glucoside isomer 1 & 20.886 & $\mathrm{C} 16 \mathrm{H} 20 \mathrm{O} 9$ & 356.1097 & 355.1097 & 193.0508 & + & + & 27 \\
\hline 14. & Ferulic acid glucoside isomer 2 & 21.840 & $\mathrm{C} 16 \mathrm{H} 20 \mathrm{O} 9$ & 356.1097 & 355.1097 & 193.0508 & - & + & 27 \\
\hline 15. & \begin{tabular}{|l|} 
Caffeic acid syryngoyl \\
O-diglucosyl ester
\end{tabular} & 21.972 & C30CH36O18 & 684.2654 & 683.2654 & 503.1987; 341.1430; 179.0558 & + & - & - \\
\hline 16. & Sesquiterpene & 22.919 & & 504.2468 & $\begin{array}{l}549.2509 \\
{\left[\mathrm{M}+\mathrm{HCOO}^{-}\right.}\end{array}$ & $\begin{array}{l}\text { 503.2468; 463.2046; 410.5768; } \\
371.1986 ; 325.0901 ; 251.0740 ; \\
149.0441\end{array}$ & + & - & - \\
\hline 17. & $\begin{array}{l}\text { Quercetin-3-O-hexose-deox- } \\
\text { yhexose }\end{array}$ & 22.406 & $\mathrm{C} 30 \mathrm{H} 26 \mathrm{O} 14$ & 609.1269 & 609.1196 & $\begin{array}{l}\text { 463.1251; 301.0352; } 179.0011 ; \\
150.9974 ; 151.0030\end{array}$ & - & + & 32 \\
\hline 18. & Hydroxybenzoic acid isomer 2 & 23.643 & $\mathrm{C} 7 \mathrm{H} 6 \mathrm{O} 3$ & 138.0227 & 137.0227 & 93.0335 & - & + & 60 \\
\hline 19. & Ferulic acid isomer & 24.282 & $\mathrm{C} 10 \mathrm{H} 10 \mathrm{O} 4$ & 194.0545 & 193.0545 & $161.0258 ; 134.0380$ & + & + & 25 \\
\hline 20. & Ferulic acid & 25.544 & $\mathrm{C} 10 \mathrm{H} 10 \mathrm{O} 4$ & 194.0545 & 193.0545 & $161.0258 ; 134.0370$ & + & + & 25 \\
\hline 21. & Ferulic acid dihexoside & 26.303 & $\mathrm{C} 22 \mathrm{H} 30 \mathrm{O} 14$ & 518.1730 & 517.1730 & 193.0517 & + & - & 28 \\
\hline 22. & $\begin{array}{l}\text { 3-O-glucopyranosyl- } \\
\text { glucopyranosyl - ara- } \\
\text { binopyranosyl-bayogenin } \\
\text { 28-O-rhamnopyranosyl(1- } \\
\text { 4)-glucopyranosyl(1-6)-glu- } \\
\text { copyranosyl ester } \\
\end{array}$ & 27.784 & $\mathrm{C} 65 \mathrm{H} 106 \mathrm{O} 33$ & 1414.6677 & 1413.6677 & $\begin{array}{l}943.4818 ; 781.4267 ; 619.3908 \\
487.3647 ; 471.2445\end{array}$ & + & - & 18,19 \\
\hline 23. & Chicoric acid isomer 1 & 28.261 & $\mathrm{C} 20 \mathrm{H} 26 \mathrm{O} 13$ & 474.0806 & 473.0806 & $\begin{array}{l}311.0349 ; 179.0360 ; 149.0092 ; \\
135.0442\end{array}$ & + & + & 29,62 \\
\hline 24. & $\begin{array}{l}\text { 3-O-glucopyranosyl- } \\
\text { glucopyranosyl - ara- } \\
\text { binopyranosyl - hederagenin } \\
\text { 28-O-rhamnopyranosyl(1- } \\
\text { 4)-glucopyranosyl(1-6)-glu- } \\
\text { copyranosyl ester }\end{array}$ & 28.375 & $\mathrm{C} 65 \mathrm{H} 106 \mathrm{O} 32$ & 1398.6615 & 1397.6515 & $\begin{array}{l}927.4645 ; 765.3851 ; 603.3728 ; \\
471.3320\end{array}$ & + & + & 18,19 \\
\hline 25. & $\begin{array}{l}\text { 3-O-glucopyranosyl-arabino- } \\
\text { pyranosyl - } \\
\text { bayogenin } \\
\text { 28-O-rhamnopyranosyl(1- } \\
\text { 4)-glucopyranosyl(1-6)-glu- } \\
\text { copyranosyl ester } \\
\end{array}$ & 28.762 & $\mathrm{C} 59 \mathrm{H} 96 \mathrm{O} 28$ & 1252.6153 & 1251.6153 & $\begin{array}{l}\text { 781.4519; 619.3788; 487.3556; } \\
471,0539 ; 469.1561\end{array}$ & + & + & 18,19 \\
\hline 26. & Chicoric acid isomer 2 & 29.091 & $\mathrm{C} 20 \mathrm{H} 26 \mathrm{O} 13$ & 474.0806 & 473.0806 & $\begin{array}{l}311.0258 ; 179.0271 ; 148.9993 ; \\
135.0311\end{array}$ & + & + & 29,62 \\
\hline 27. & $\begin{array}{l}\text { Pulsatilloside D (3-O-glu- } \\
\text { copyranosyl-arabinopyranosyl } \\
- \\
\text { 23-hydroxybetulinic acid } \\
\text { 28-O-rhamnopyranosyl(1- } \\
\text { 4)-glucopyranosyl(1-6)-glu- } \\
\text { copyranosyl ester) }\end{array}$ & 29.407 & $\mathrm{C} 59 \mathrm{H} 96 \mathrm{O} 27$ & 1236.6207 & 1235.6207 & $\begin{array}{l}765.4164 ; 603.3685 ; 471.1480 \\
469.1372 ; 451.1337\end{array}$ & + & + & $18,19,21$ \\
\hline 28. & \begin{tabular}{|l|} 
3-O- arabinopyranosyl - \\
bayogenin \\
28-O-rhamnopyranosyl(1- \\
4)-glucopyranosyl(1-6)-glu- \\
copyranosyl ester
\end{tabular} & 29.899 & $\mathrm{C} 53 \mathrm{H} 86 \mathrm{O} 23$ & 1090.5632 & 1089.5632 & $\begin{array}{l}\text { 619.3765; 473.4086; 471.1748; } \\
469.1635 ; 478.4177\end{array}$ & + & + & 18,19 \\
\hline 29. & $\begin{array}{l}\text { Leonloside D (3-O-glucopyra- } \\
\text { nosyl-arabinopyranosyl - } \\
\text { hederagenin } \\
\text { 28-O-rhamnopyranosyl(1- } \\
\text { 4)-glucopyranosyl(1-6)-glu- } \\
\text { copyranosyl ester) }\end{array}$ & 30.282 & $\mathrm{C} 59 \mathrm{H} 96 \mathrm{O} 27$ & 1236.6207 & 1235.6207 & $\begin{array}{l}\text { 765.4365; 603.3756; 471.1528; } \\
469.1538 ; 451.1649\end{array}$ & + & + & $14,18,19$ \\
\hline \multicolumn{10}{|l|}{ Continued } \\
\hline
\end{tabular}




\begin{tabular}{|c|c|c|c|c|c|c|c|c|c|}
\hline Comp. No & Tentative identification & Rt (min) & Molecular fromula & MW & {$[\mathrm{M}-\mathrm{H}]^{-}$} & Fragments $(\mathbf{m} / \mathbf{z})$ & Roots & Leaves & References \\
\hline 30. & $\begin{array}{l}\text { 3-O-arabinopyranosyl - } \\
\text { 23-hydroxybetulinic acid } \\
\text { 28-O-rhamnopyranosyl(1- } \\
\text { 4)-glucopyranosyl(1-6)-glu- } \\
\text { copyranosyl ester }\end{array}$ & 31.265 & $\mathrm{C} 53 \mathrm{H} 86 \mathrm{O} 22$ & 1074.5681 & $\begin{array}{l}1119.5616 \\
{\left[\mathrm{M}+\mathrm{HCOO}^{-}\right.}\end{array}$ & $\begin{array}{l}\text { 1073.5681; 603.3960; } \\
471.11869 ; 469.1579 ; 409.1282\end{array}$ & + & + & $18,19,22$ \\
\hline 31. & Coumaric acid derivative & 32.635 & $\mathrm{C} 21 \mathrm{H} 28 \mathrm{O} 13$ & 488.0960 & 487.0960 & $325.0578 ; 163.0298$ & + & + & - \\
\hline 32. & $\begin{array}{l}\text { Hederacoside C } \\
\text { (3-O-arabinopyranosyl- rham- } \\
\text { nopyranosyl } \\
\text { hederagenin } \\
\text { 28-O-rhamnopyranosyl(1- } \\
\text { 4)-glucopyranosyl(1-6)-glu- } \\
\text { copyranosyl ester) } \\
\text { / Anemoside B4 } \\
\text { (3-O- arabinopyranosyl- rham- } \\
\text { nopyranosyl } \\
\text { 23-hydroxybetulinic acid } \\
\text { 28-O-rhamnopyranosyl(1- } \\
\text { 4)-glucopyranosyl(1-6)-glu- } \\
\text { copyranosyl ester) }\end{array}$ & 33.025 & $\mathrm{C} 59 \mathrm{H} 96 \mathrm{O} 26$ & 1220.6263 & 1219.6263 & 749.4374; 471.1673; 469.1582 & + & - & $18,21,22$ \\
\hline 33. & $\begin{array}{l}O \text { - arabinopyranosyl - glu- } \\
\text { copyranosyl } \\
\text { 23-hydroxybetulinic acid ester }\end{array}$ & 33.510 & $\mathrm{C} 41 \mathrm{H} 66 \mathrm{O} 13$ & 766.4445 & $\begin{array}{l}811.4601 \\
{\left[\mathrm{M}+\mathrm{HCOO}^{-}\right.}\end{array}$ & $\begin{array}{l}765.4445 ; 603.3898 ; 585.3713 \\
453.3383\end{array}$ & + & - & 18,19 \\
\hline 34. & Dehydrodiferulic acid isomer 1 & 33.571 & $\mathrm{C} 20 \mathrm{H} 18 \mathrm{O} 8$ & 386.0992 & 385.0992 & 193.0515; 161.0237; 134.0391 & - & + & 58,26 \\
\hline 35. & \begin{tabular}{|l|} 
3-O- arabinopyranosyl - \\
hederagenin \\
28-O-rhamnopyranosyl(1- \\
4)-glucopyranosyl(1-6)-glu- \\
copyranosyl ester
\end{tabular} & 33.889 & $\mathrm{C} 53 \mathrm{H} 86 \mathrm{O} 22$ & 1074.5681 & 1073.5681 & $\begin{array}{l}\text { 603.3825; 471.1534; 469.1557; } \\
409.1250\end{array}$ & + & - & 18,19 \\
\hline 36. & $\begin{array}{l}\text { Pulsatilloside C } \\
\text { (28-O-rhamnopyranosyl(1- } \\
\text { 4)-glucopyranosyl(1-6)-glu- } \\
\text { copyranosyl 23-hydroxybetu- } \\
\text { linic acid ester) }\end{array}$ & 34.334 & $\mathrm{C} 48 \mathrm{H} 78 \mathrm{O} 18$ & 942.5085 & $\begin{array}{l}987.5190 \\
{\left[\mathrm{M}+\mathrm{HCOO}^{-}\right.}\end{array}$ & $941.5085 ; 471.3455 ; 469.1608$ & + & + & 22,63 \\
\hline 37. & $\begin{array}{l}\text { 3-O- arabinopyranosyl - glu- } \\
\text { copyranosyl } \\
\text { 23-hydroxybetulinic acid ester }\end{array}$ & 35.472 & $\mathrm{C} 41 \mathrm{H} 66 \mathrm{O} 13$ & 766.4527 & $\begin{array}{l}811.4601 \\
{\left[\mathrm{M}+\mathrm{HCOO}^{-}\right.}\end{array}$ & $\begin{array}{l}\text { 765.4527; 603.3868; 585.3713; } \\
\text { 453.3383; }\end{array}$ & + & - & 18,19 \\
\hline 38. & \begin{tabular}{|l|} 
23-hydroxybetulinic acid \\
O-rhamnopyranoside
\end{tabular} & 36.694 & $\mathrm{C} 36 \mathrm{H} 58 \mathrm{O} 8$ & 618.1762 & 617.1762 & $471.1239 ; 453.1241 ; 437.0878$ & + & - & 18,19 \\
\hline 39. & Dehydrodiferulic acid isomer 2 & 38.050 & $\mathrm{C} 20 \mathrm{H} 18 \mathrm{O} 8$ & 386.0992 & 385.0992 & $193.0515 ; 161.0237 ; 134.0391$ & - & + & 26 \\
\hline 40. & $\begin{array}{l}\begin{array}{l}\text { Hederagenin } O \text {-rhamnopyra- } \\
\text { noside }\end{array} \\
\text { - }\end{array}$ & 38.484 & $\mathrm{C} 36 \mathrm{H} 58 \mathrm{O} 8$ & 618.1762 & 617.1762 & $471.1239 ; 453.1241 ; 437.0878$ & + & - & 18,19 \\
\hline 41. & $\begin{array}{l}\text { 3-O- arabinopyranosyl - 28-O- } \\
\text { glucopyranosyl } \\
\text { hederagenin ester }\end{array}$ & 39.864 & $\mathrm{C} 41 \mathrm{H} 66 \mathrm{O} 13$ & 766.4403 & $\begin{array}{l}811.4601 \\
{\left[\mathrm{M}+\mathrm{HCOO}^{-}\right.}\end{array}$ & $765.4403 ; 603.3916 ; 471.3386$ & + & - & 18,19 \\
\hline 42. & $\begin{array}{l}\text { 3-O- arabinopyranosyl - 28-O- } \\
\text { glucopyranosyl } \\
\text { hederagenin ester }\end{array}$ & 40.361 & $\mathrm{C} 41 \mathrm{H} 66 \mathrm{O} 13$ & 766.4434 & $\begin{array}{l}811.4601 \\
{\left[\mathrm{M}+\mathrm{HCOO}^{-}\right.}\end{array}$ & $765.4434 ; 603.3794 ; 471.3386$ & + & + & 18,19 \\
\hline 43. & Unknown & 49.457 & & 783.5296 & 782.5296 & $\begin{array}{l}\text { 615.4189; 573.3723; 539.9341; } \\
355.2680\end{array}$ & + & + & \\
\hline
\end{tabular}

Table 4. Chemical composition of $P$. patens extracts.

to be hederagenin esters with various glucose location (Fig. 4). According to Jin et al. ${ }^{18}$ one of the present isomers 41 or 42 could be tentatively assigned as Plusatilla B (3-O-arabinopyranosyl-28-O-glucopyranosyl hederagenin ester) previously reported in $P$. chinensis roots.

Compound 36 formed two ion peaks at $\mathrm{m} / z 987$ and at $\mathrm{m} / z$ 941, assigned as adduct ion [M+HCOO] ${ }^{-}$and precursor ion $[\mathrm{M}-\mathrm{H}]^{-}$, respectively. Further fragmentation yielded characteristic ion at $m / z 471$, as a result of the typical neutral loss of $470 \mathrm{Da}$, suggesting that C-28 position was substituted with Glu-Glu-Rha sugar chain. The same fragmentation patterns as in literature data allowed compound $\mathbf{3 6}$ to be identified as 23 -hydroxybetulinic acid 28-O-rhamnopyranosyl(1-4)-glucopyranosyl(1-6)-glucopyranosyl ester, known as Pulsatilloside $\mathrm{C}^{22}$.

Compound 32 characterized with precursor ion at $\mathrm{m} / z 1219$ and two notable fragment ions at $\mathrm{m} / z 749$ and 603 , showed a decrease of $146 \mathrm{Da}$ compared to that of saponin 30, indicating the presence of one Rha moiety $(146 \mathrm{Da})$ in $\beta$-sugar chain. The sequence of sugar moieties linked to C-3 position was determined based on literature data ${ }^{23}$. The characteristic product ion at $\mathrm{m} / z 749$ might indicate on Anemoside B4 structure also found in P. chinensis and P. korean ${ }^{21-23}$. However, in the absence of sufficient data, the compound 32 might be as well tentatively identified as Olean-type saponin, namely Hederacoside $C^{18}$.

Another group of secondary metabolites identified in samples of $P$. patens were phenolic acids. In the category of hydroxycinnamic acid the most abundant were caffeic and ferulic acids derivatives, which occurrence was in accordance with data reported for P. pratensis (L.) Mill by ${ }^{24}$.

Compound 20 presented the precursor ion $[\mathrm{M}-\mathrm{H}]^{-}$at $\mathrm{m} / z 193$ and yielded the fragment ions at $\mathrm{m} / z 178$ and 134 , attributed to the cleavage of $\mathrm{CH}_{3}$ group and characteristic for phenolic acids loss of carbon dioxide from the carboxylic acid group ${ }^{25}$. By comparison with the UV-vis spectra, retention time and fragments generated 


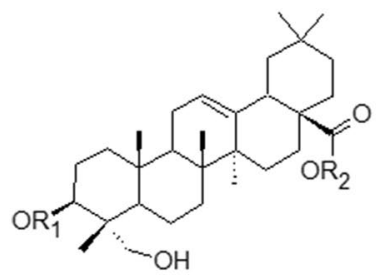

HED

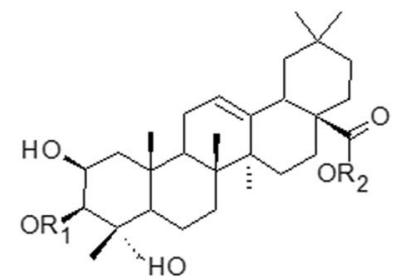

BAY

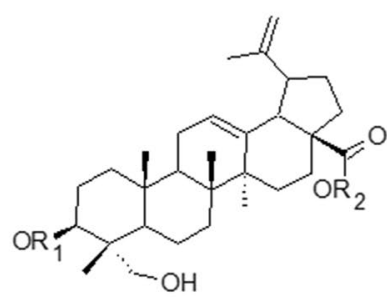

BET

$\begin{array}{cccc}\text { Compound } & \text { Basic skeleton } & R_{1} & R_{2} \\ 24 & \text { HED } & \text { Ara-Glc-Glc } & \text { Glc-Glc-Rha } \\ 27 & \text { BET } & \text { Ara-Glc } & \text { Glc-Glc-Rha } \\ 29 & \text { HED } & \text { Ara-Glc } & \text { Glc-Glc-Rha } \\ 30 & \text { BET } & \text { Ara } & \text { Glc-Glc-Rha } \\ 35 & \text { HED } & \text { Ara } & \text { Glc-Glc-Rha } \\ 38 & \text { BET } & & \text { Rha* } \\ 40 & \text { HED } & & \text { Rha* } \\ 32 & \text { HED/BET } & \text { Ara Rha } & \text { Glc-Glc-Rha } \\ 33 & \text { BET } & \text { Ara } & \text { Glc } \\ 37 & \text { BET } & \text { Ara } & \text { Glc } \\ 41 & \text { HED } & \text { Ara } & \text { Glc } \\ 42 & \text { HED } & \text { Ara } & \text { Glc } \\ 36 & \text { BET } & & \text { Glc-Glc-Rha } \\ 22 & \text { BAY } & \text { Ara-Glc-Glc } & \text { Glc-Glc-Rha } \\ 25 & \text { BAY } & \text { Ara-Glc } & \text { Glc-Glc-Rha } \\ 28 & \text { BAY } & \text { Ara } & \text { Glc-Glc-Rha } \\ 0^{c-3} \text { or c-28 position } & & & \end{array}$

Figure 4. Types of triterpenoid saponins identified in P. patens extracts.

during CID of the reference standard, compound 20 was unambiguously identified as ferulic acid, while peak 19 was its isomer.

The remaining ferulic acid derivatives were tentatively assigned as ferulic acid glucoside (isomers 13 and 14) or dihexoside (compound 21) due to the neutral loss of one or two hexoside moieties from $[\mathrm{M}-\mathrm{H}]^{-}$resulting in an abundant fragment ion at $m / z$ 193. Similar fragmentation pattern observed for compounds 11, 13, 14, 21, 34, 39 indicated on ferulic acid in their structure. Based on work ${ }^{26}$ compounds 34 and 39 were proposed to be isomers of dehydrodiferulic acids.

The mass spectrum for compound 8 showed the precursor ion $[\mathrm{M}-\mathrm{H}]^{-}$at $\mathrm{m} / z 179$ and was identified as caffeic acid. Other typical for caffeic acid ions at $m / z 135$ and 161, derived from the decarboxylation and dehydration of caffeic acid as well as ions at $m / z 107$ seen in MS/MS spectrum, was found to be consistent with previously published data ${ }^{27,28}$. The remaining compounds $\mathbf{1}, \mathbf{5}, \mathbf{1 5}, \mathbf{2 3}$ and 26 were tentatively identified as caffeic acid derivatives, according to their MS/MS spectral data. For instance, compounds $\mathbf{1}$ and $\mathbf{5}$ generated similar pseudo molecular ion at $\mathrm{m} / z$ 341. The neutral loss of hexoside moiety $(-162 \mathrm{Da})$ resulted in characteristic for caffeic acid, fragment ion at $m / z 179$. It must be noted that the loss of $162 \mathrm{Da}$ moiety may also result from the neutral loss of single caffeic acid molecule ${ }^{29}$. Thus, compound $\mathbf{1}$ was tentatively assigned as caffeic acid hexoside while compound 5, which generates similar fragment ions, as dicaffeic acid ${ }^{30}$. According to fragments observed in MS/ MS spectrum of compound 15 such as ions at $m / z 683,503,341$ and 179 this molecule was tentatively identified as caffeic acid syryngoyl O-diglucosyl ester.

Another pair of compounds, 23 and 26 producing the same precursor ion $[\mathrm{M}-\mathrm{H}]^{-}$at $\mathrm{m} / \mathrm{z} 473$ with a molecular formula of $\mathrm{C}_{20} \mathrm{H}_{26} \mathrm{O}_{13}$ was identified. Their MS/MS spectrum revealed the prominent ion at $m / z 311$, due to the neutral loss of a single caffeic acid molecule $(-162 \mathrm{Da})$ and diagnostic fragment ions at $\mathrm{m} / z 179$ (caffeic acid), 149 
(tartaric acid) as well as $m / z$ 135, corresponding to caffeic acid decarboxylation. Because of lack of product ion at $m / z 341$ (relevant for caftaric acid glycoside) in both MS/MS spectra and based on the work ${ }^{29}$, the compounds 23 and $\mathbf{2 6}$ could be classified as isomers of chicoric acid (=di-caffeoyltartaric acid).

Similarly, compound 7 characterized by precursor ion at $m / z 311$ and a molecular formula of $\mathrm{C}_{13} \mathrm{H}_{12} \mathrm{O}_{9}$, was tentatively identified as caftaric acid. The tree notable product ions observed in MS/MS spectrum at $\mathrm{m} / z 179$, 149 and 135 were in accordance to data reported by ${ }^{29,30}$ for other plant materials.

Among hydroxycinnamic acids, trace amounts of coumaric acid primarily in roots and its derivatives (in leaves) were also found. Compound 6 yielded precursor ion at $m / z 163$ and a molecular formula of $\mathrm{C}_{9} \mathrm{H}_{8} \mathrm{O}_{3}$. The most prominent product ions were at $\mathrm{m} / z 135(-28 \mathrm{Da})$ and $119(-44 \mathrm{Da})$. Regarding presented data, peak 6 was presumed to be coumaric acid. Similarly, for compound 31 the fragmentation of $[\mathrm{M}-\mathrm{H}]^{-}$at $\mathrm{m} / z 487$ produced two notable product ions at $m / z 325$ and at $m / z$ 163. Based on this data compound 31 was tentatively identified as coumaric acid derivative.

The ESI-MS signals for hydroxybenzoic acid derivatives were also identified in analyzed samples, mainly at $\mathrm{m} / z 167,181$ and 137, corresponding to valinic (10), hydroxymelilotic (3) and hydroxybenzoic acids isomers (4 and 18), respectively. Compared with literature data reported by ${ }^{30}$ and spectrum obtained from Human Metabolome Database the fragmentation ions at $\mathrm{m} / z 152$ and 108 confirmed the valinic acid structure. In terms of compounds 4 and 18 further fragmentation by CID, revealed the characteristic loss of $\mathrm{CO}_{2}$ from its precursor ion, generating the diagnostic ion at $\mathrm{m} / z$ 93. Due to the identical fragmentation pattern, we deduced compounds 4 and 18 to be isomers of hydroxybenzoic acid. Compound 2 characterized by precursor ion at $\mathrm{m} / z 315$ and fragment ions at $m / z 153,109$ was tentatively identified as dihydroxybenzoic acid hexoside, by comparison with spectral data reported for other plant samples by ${ }^{27,31}$.

Compound 17 generated product ions at $\mathrm{m} / z 463$ and 301 from precursor ion $[\mathrm{M}-\mathrm{H}]^{-}$at $\mathrm{m} / \mathrm{z} 608$. It's characteristic UV-vis spectra $\left(\lambda_{\max } 257,357 \mathrm{~nm}\right)$ as well as detected in MS/MS spectrum diagnostic fragment ions at $m / z 179$ and 151 supported the existence of a quercetin aglycone. So the chemical structure of compound 17 was tentatively identified as quercetin-3-O-hexose-deoxyhexose ${ }^{32}$.

According to ${ }^{33}$ the anemonin structure was also reported in the roots of $P$. patens. The peak 9 was characterized with a molecular formula of $\mathrm{C}_{10} \mathrm{H}_{8} \mathrm{O}_{4}$ and fragment ions at $m / z 191,147$ and 101 .

Unfortunately, some of the constituents could not be identified 12, 43 and 18 (sesquiterpene) because of the insufficient data.

\section{Discussion}

Uncontrolled cell division, escaping the external and internal control of the cell cycle, leads to unlimited proliferation (multiplication) of cancer cells and neoplastic processes (neoplasia, carcinogenesis) ${ }^{34}$. This is often due to mutations in DNA regions encoding genes important for cell cycle control mechanisms. A panel of 13 vectors (transcription factors) induced by the luciferase reporter gene was used in our research. These vectors enable efficient expression of the protein encoded by the luciferase reporter gene in the system under investigation. Their expression was carried out by enhancer elements (inducers) (such as, IL6, TGF- $\beta$, PMA, Wnt 3a) that bind to specific transcription factors. The vectors and inducers included in this study and the studies of other authors $^{17,35}$ are used to assess the activity of most of the cancer-related signaling pathways. Similarly [(E)-3,5, $4^{\prime}-$ trihydroxystilbene], a parent compound of the analog used in this study, which is present in a wide range of plants, such as Arachis hypogaea L. (Fabaceae) or Vitis vinifera L. (Vitaceae), is used as a positive control ${ }^{35}$. It is the type of natural phenol, and a phytoalexin produced by several plants in response to injury or when the plant is under attack by pathogenic bacteria and fungi. Resveratrol is the most well-known stilbene, which is postulated as a potential modulator of signal transduction pathways for cancer and the carcinogen response ${ }^{35}$.

The modulation of cancer-related signaling pathways and determining the mechanism of action of transcription factors on the inhibition of the neoplastic process is an important topic in recent years.

STAT3 (Signal Transducers and Activators of Transcription) is a protein, involved in the transmission and the activation of gene transduces from the STAT protein family. In the Stat3 signaling pathways, cytokine IL-6 used as an inducer in our research leads to its activation (Janus kinase JAK) (Fig. 3). Stat3 is phosphorylated by the receptor ${ }^{36}$ and translocated to the nucleus, where it promotes transcription of target genes, including prooncogenetic ones ${ }^{37}$. The inhibition of STAT3 in HeLa cell signaling caused by methanolic extract of $P$. patens is associated with the inhibition of growth and inhibition of cell proliferation and their apoptosis (Fig. 3).

Smad is a signaling pathway activated by TGF- $\beta$ and taking part in the regulation of target gene expression (Fig. 3). In our study, in Smad/ TGF- $\beta$ signaling pathway, the ligand TGF- $\beta$ was bound and connected with two pairs of type I and type II receptors, which are transmembrane Ser/Thr kinases. In these receptor complexes, the type II receptor phosphorylated and activated the type I receptor kinase, which then phosphorylated and activated Smad. The Smad/TGF- $\beta$ signal transduction pathway is important in signaling and regulation of cells functions during the whole life of the organism ${ }^{38}$.

The MYC-is an induced nuclear protein antigen, encoded by the MINA gene. MYC is a transcription factor involved in proliferation, growth, and cellular development, and direct regulation of target genes. MYC is controlling the complex networks of microRNAs and apoptosis mediators ${ }^{39}$. In this study, MYC was activated by PMA (protein kinase activator), which is phorbol 12-myristate-13-acetate (Fig. 3). As reported previously by several researchers, MYC can be activated by the direct interaction with the acetyltransferases p300 and CBP ${ }^{39}$. In our modulation of cancer-related signaling pathways, many transcription factors were activated by PMA (protein kinase activator). These are NF- $\kappa \mathrm{B}$ (nuclear factor kappa-light-chain-enhancer of activated B cells), AP-1 (Activator Protein 1), Ets, Hedgehog, and Notch (Fig. 3). NF- $\kappa$ B can also be activated by the enzyme I $\kappa B$ kinase and then translocated into the nucleus. The Notch can be activated by specific Serine/threonine (Ser/ Thr) protein kinase and E2F by-Cyclins, cyclin-dependent kinases CDKs connected with the cyclin-dependent 


\begin{tabular}{|c|c|c|c|}
\hline Name of the vector & Inducer (Alternate inducer) & Fold of induction & Duration of treatment \\
\hline STAT3 & IL6 & $15 \mathrm{x}$ & \multirow{8}{*}{ 4-h induction } \\
\hline SMAD3/4 & TGF- $\beta$ & $15 \mathrm{x}$ & \\
\hline $\mathrm{NF}-\kappa \mathrm{B}$ & PMA & $20 x$ & \\
\hline AP-1 & PMA & $50 \mathrm{x}$ & \\
\hline Ets & PMA & $8 \mathrm{x}$ & \\
\hline E2F & PMA & $4 \mathrm{x}$ & \\
\hline MYC & PMA & $7 \mathrm{x}$ & \\
\hline pTK control & No inducer & Not applicable & \\
\hline Notch & PMA & $4 \mathrm{x}$ & \multirow{6}{*}{ 6-h induction } \\
\hline FoxO & No inducer & Not applicable & \\
\hline Wnt & Wnt-3a & $4 \mathrm{x}$ & \\
\hline Hedgehog & PMA & $20 \mathrm{x}$ & \\
\hline miR-21 & No inducer & Not applicable & \\
\hline $\mathrm{k}$-Ras & No inducer & Not applicable & \\
\hline
\end{tabular}

Table 5. The panel of 13 inducible luciferase reporter gene vectors where expression is driven by enhancer elements (Inducer) that bind to specific transcription factors.

kinase inhibitors ( $\mathrm{p} 15, \mathrm{p} 16)$. This article reports the use of the methanolic extract of the $P$. patens which inhibits the progression of cancer through the modulation of cancer-related metabolic signaling pathways. Numerous studies support a pro-oncogenic function for Notch signaling too. It was reported that the modulation of the Notch pathway is important in controlling the development of cancer cells and has bidirectional complicated interaction with multiple other pathways that include candidate therapeutic targets ${ }^{40}$. Same for the Ets family (Erythroblast Transformation Specific) which are one of the largest families of transcription factors to be associated with cancer, such as through gene fusion ${ }^{41}$. Their deregulation following changes in expression and transcriptional activity plays a critical role in carcinogenesis (neoplasm, tumor formation), apoptosis (programmed cell death), and neoplastic angiogenesis by the ability to form blood vessels within a neoplastic tumor enables further growth tumor mass. All ETS family members are identified through a highly conserved DNA binding domain, the ETS domain, which is a winged helix-turn-helix structure that binds to DNA sites with a central GGA (A/T) DNA sequence ${ }^{38}$.

The E2F family comprises of basic helix-loop-helix transcription factors that supervise the expression of genes associated with the regulation of the cell cycle ${ }^{42}$. The transcriptional targets of E2F include cyclins, cyclindependent kinases, checkpoints regulators, DNA repair, and replication proteins (Fig. 3). The cancer-related proliferative roles of E2F family members represent a recent evolutionary adaptation ${ }^{42}$. The evolutionarily conserved Hedgehog (Hh) signaling pathway (synonyms: Hedgehog-Patched (Hh-Ptch), Hedgehog-Patched-Smoothened (Hh-Ptch-Smo)) is responsible for signal conveyance from the cell membrane into the nucleus (Fig. 3). The Hh signaling pathway plays a significant role in normal embryonic development and the maintenance of stem cells important for tissue repairs, such as mammary, skin, neural, lung stem cells, and some epithelial cells of internal organs ${ }^{43}$.

Activator protein 1 (AP-1) is a transcription factor responsible for the regulation of gene expression in reaction to various stimulants, including viral and bacterial infections, growth factors, cytokines and stress. AP-1 controls proliferation, differentiation and apoptosis ${ }^{44}$. A variety of extracellular matrix and genotoxic agents were shown to induce AP-1 activity, which supports the involvement of AP-1 in programmed cell death.

The FoxO transcription factors oversee the protection from oxidative stress by regulating antioxidants and controlling the protein quality. If the expression or activation of FoxO becomes dysregulated it may lead to a variety of age-related disorders affecting CNS (central nervous system) bones, muscles, or leading to cancer ${ }^{45}$. Moreover, the FoxO regulates the cell cycle, the proliferation of cells and apoptosis.

Protein Wnt-3a is encoded by the WNT3A gene ${ }^{46}$ which belongs to the WNT gene family. The WNT includes a series of genes that encode secreted signaling proteins (Fig. 3) which are involved in adipogenesis, oncogenesis, and the regulation of embryogenesis. WNT3 and WNT3A genes are two human paralogues of mouse protooncogene Wnt3, which induces carcinogenesis through activation of the $\beta$-catenin-TCF signaling pathway ${ }^{46}$.

Reporter genes were introduced by transfection into cells grown in cell culture (Table 5). The inhibition of the progression of human cancer cells by many cancer-related signaling pathways were studied on the species Pulsatilla patens (L.) Mill. The Pulsatilla species is a good candidate as a natural product for use in the treatment of cancer. This is indicated by the research of other authors about $P$. koreana Nakai ${ }^{16}$ or P. chinensis (Bunge) Regel $^{47}$. In the study with P. koreana it was found that SB365, Pulsatilla saponin D isolated from the root of this species, strongly suppressed the growth and proliferation of colon cancer cells and induced their apoptosis. Additionally, SB365 showed antiangiogenic activity and significantly inhibited tumor growth ${ }^{16}$. In anticancer mechanism, SB365 effectively suppressed the AKT/mTOR pathway both in vitro and in vivo. Moreover, deoxypodophyllotoxin (DPT) from the extract of $P$. koreana was found as a cytotoxic and antiangiogenic component combined with antitumor activity. The DPT from the extract of Pulsatillae Radix which exhibited antiangiogenic activity and good antitumor activity against mice bearing Lewis lung carcinoma (LLC) is an active constituent of $\mathrm{SB} 31^{48}$. The biologically active compounds isolated from the root extract of $P$. koreana have been found to show 
antibacterial, antiparasitic and anti-inflammatory ${ }^{49}$ activities too. Other species $P$. patens and $P$. vulgaris from our previous research showed antibacterial and antifungal activities ${ }^{50}$. P. chinensis has been found to show antitumor activities too. Pulsatilla saponin A from this species significantly inhibited the growth of human hepatocellular carcinoma SMCC-7721 cells and pancreatic BXPC3 and SW1990 cancer cells ${ }^{47}$. Similar inhibitory activities were observed when the compound was tested in mouse xenograft tumor models using human hepatocellular carcinoma Bel-7402 and pancreatic cancer SW1990 cells. Pulsatilla saponin A may exert its antitumor effect by inducing DNA damage and causing G2 arrest and apoptosis in cancer cells ${ }^{47}$. The triterpene glycosides (Pulsatilla saponins $\mathrm{A}$ and $\mathrm{B}$ ) and the triterpene acid isolated from $P$. chinensis shows high cytotoxic activity against the malignant cells of the lung cancer ${ }^{21}$ and are used for antibacterial, antiparasitic, antiprotozoal, antifungal and molluscicidic ${ }^{51}$ activities. Our study about the active compounds of methanolic extracts of $P$. patens is very important too because these are the first studies of Pulsatilla patens species which its antitumor properties in the luciferase reporter test performed.

There are various classes of plant secondary metabolites influencing cancer-related signaling pathways. Triterpenoid saponins, naphthoquinones, chalcones, sesquiterpene lactones, curcuminoids, flavonoids and isothiocyanates are selective for cancer cells because they are much more active within cancer cells than in normal cells (Supplementary Table S2). Transformed cells are less able to handle the oxidative stress induced by these compounds and therefore redox-sensitive targets are more greatly impacted. These classes of compounds are great for targeting cancer-relevant signaling pathways in cancer cells and play a major role in reactive oxygen species (ROS) generation ${ }^{52}$, the depletion of the antioxidant glutathione (GSH), target redox reactions, and redox-sensitive cysteine ${ }^{53}$.

The Authors hereby would like to point out the limitation related to the presented results. The suggested influence of $P$. patens on apoptosis was based on the studies of cancer-related signaling pathways in HeLa cells, being the main goal of our research, and not on the direct measurements of apoptosis. We acknowledge that the potential proapoptotic activity of $P$. patens requires additional research focusing on apoptosis with the use of direct measurement of cellular caspases (caspase-3, -7, -8 or -9), DNA fragmentation, and Annexin V based assays using fluorescence microscopy or flow cytometry.

Our phytochemical analysis of the methanolic extracts of the $P$. patens shows that compounds, which strongly suppressed the growth and proliferation of HeLa cancer cells are mainly saponins. The triterpenoid saponins and phenolic acids were characterized and identified from a methanolic extract of the $P$. patens roots and leaves. Similar biological properties of saponins of the genus Pulsatilla in the treatment of cancer and other diseases together with $P$. korean $a^{54}$ and $P$. chinensis ${ }^{47}$ has been shown for other species such as P. cernua (Thunb.) Bercht. et Opiz. Too ${ }^{55}$, P. nigricans Storck ${ }^{56}$, . dahurica (Fisch. ex DC.) Spreng. ${ }^{57}$, P. turczaninovii Kryl. et Serg. ${ }^{58}$ or $P$. pratensis (L.) Mill. ${ }^{24}$. Saponins from Pulsatilla spp. have demonstrated multiple biological properties including antitumor ${ }^{59}$, neuroactive, immunomodulatory, antioxidant, cognition-enhancing and neuroprotective ${ }^{55}$ activities. In our research was found that active compounds resulted in inhibition of cell growth/proliferation and induction of apoptosis of HeLa cells-cervical cancer. The saponins isolated from the methanolic extract of $P$. patens subsp. multifida (G.A. Pritel) Zämelis, the native species from the USA and Asia, have been used for ages in traditional medicine as the agent showing anticancer properties, inhibiting the growth of skin cancer ${ }^{20}$. But no one until now has identified the antitumor properties of $P$. patens subsp. patens of a native species from Europe using cancer-related signaling pathways in HeLa cells.

\section{Conclusion}

Research on the methanolic extracts of $P$. patens confirms strong inhibition of signaling pathways in HeLa cells, a cervical cancer cell line, which may also be of importance for the chemotherapeutic ability in other types of cancer. A characteristic feature of active compounds from $P$. patens is their activity at many levels of cell signaling. In this study it was found, that the root extract of $P$. patens is the most potent in inhibiting the activation of Stat3, Smad, AP-1, NF- $\kappa$ B, MYC, Ets, Wnt, and Hdghog. The pleiotropic nature of this extract is in line with current trends in oncological pharmacology, where it is to replace drugs, in favor of multidirectional therapies. The methanolic extracts of $P$. patens enhanced apoptotic death of HeLa cells, deregulated cellular proliferation, differentiation, and progression towards the neoplastic phenotype by altering key signaling molecules required for cell cycle progression. Since the proapoptotic activity of $P$. patens was observed during studies of cancer-related signaling pathways in HeLa cells the future studies should be focused on the detailed analysis of apoptosis using alternative methods, ex. direct measurement of cellular caspases. This is the first study to report the influence of Pulsatilla species on cancer signaling pathways. Effects of the methanolic extracts of $P$. patens in these signaling pathways identified here should be further evaluated in tumor-bearing mice and in the next step in preclinical studies of cervical cancer patients, treated with active compounds from this species. The results showed that $P$. patens is a rich source of polyphenolic constituents, mainly saponins, and hydroxycinnamic acids, wherein their triterpenoid saponins showed a different profile in comparison to other species from the Ranunculaceae family. Our research will be helpful to determine the relevance of each cancer-related signaling pathways that may be used to develop novel therapies that combine extracts of $P$. patens with other agents including STAT, SMAD, AP-1, NF- $\kappa B$, MYC, Ets, Wnt, and Hdghog blockers, to effectively treat cervical cancer, and other cancers that utilize these pathways.

\section{Methods}

Plant material. The leaves and roots of P. patens were collected in May 2016-2018 at the Knyszynska Forest, Podlaskie Province, in North-Eastern Poland. Plant material was identified by Prof. Grażyna Łaska from the Bialystok University of Technology. In Poland, species P. patens has been strictly protected since 1958 and still requires active protection. Collection of plant material for testing was done in accordance with Decision 
No WPN.6400.23.2016.MW of 27.04.2016 issued by the Regional Directorate of Environmental Protection in Bialystok, 23 Dojlidy Fabryczne Street, 15-554 Bialystok. In order to obtain a larger amount of plant material for future studies, a cooperation agreement was signed between the Bialystok University of Technology and Botanical Garden "Herbal Corner". Within this collaboration, whole plant species were transferred from the natural environment to the Botanical Garden for their propagation. . The use of plants parts in the present study complies with international, national guidelines. The plant material in the form of dry roots ( $23.6 \mathrm{~g})$ and leaves $(32.3 \mathrm{~g})$ was extracted by accelerated solvent extraction (ASE) technique (SpeedExtractor E-916, Buchi) with $80 \%$ methanol. The extraction time was $30 \mathrm{~min}$., the temperature was $100^{\circ} \mathrm{C}$ and the pressure was 120 bar. Three extracts were prepared from each raw material. Extracts were filtered through paper filters. Methanol was dried using a rotor evaporator. The obtained extracts were subjected to detailed phytochemical analysis.

Preparation of reference compounds solutions. To provide the quantitative analysis of saponins and hydroxycynnamic acid type compounds two reference substances, pulsatilla saponin D and ferulic acid were used. The pulsatilla saponin D was obtained from TRC-Canada (Toronto, Canada) with $90 \%$ purity, while ferulic acid was purchased from Sigma-Aldrich (Madrid, Spain). All standards were precisely weighed and individually dissolved in methanol HPLC-grade (J.T. Baker, Deventer, The Netherlands) to achieve stock solutions of $1 \mathrm{mg} /$ $\mathrm{mL}$ and stored at $4^{0} \mathrm{C}$.

Conditions for Qualitative and Quantitative LC-ESI-QTOF-MS analysis. The LC-ESI-QTOF-MS analysis was done on Agilent 1200 Infinity HPLC coupled to Agilent 6530BQTOF Accurate-Mass QTOF system equipped with Dual Agilent Jet Stream spray source (ESI) (Agilent Technologies, Santa Clara, CA, USA) and operated with $\mathrm{N}_{2}$ generator (Parker Hannifin Corporation, Haverhill, MA; generating a nitrogen supply at purities > 99\%). For the chromatographic separation Gemini column ( $3 \mu \mathrm{m}$ i.d. C18 with TMS end-capping, $110 \AA$, $100 \times 2 \mathrm{~mm}$ ) supported with a guard column (PhenomenexInc, Torrance, CA, USA) was used. Chromatographic and spectrometric conditions followed our previous report ${ }^{31}$. The MS spectra acquired in the negative mode provided greater molecular ion abundances and better sensitivity compared to the positive-ion mode. Compounds were tentatively identified based on fragmentation patterns and supported by comparison of obtained mass spectra with mass spectra obtained for reference compounds, mass spectra found in the literature and records available in Metlin database (https://metlin.scripps.edu). Quantification was done based on the UV spectra recorded in 210 and $320 \mathrm{~nm}$ for saponins and hydroxycynnamic acid type compounds, respectively.

Method validation. Calibration curves, the limit of detection and quantification. The method linearity was checked by evaluation of the dilutions prepared from stock solutions of reference substances. The construction of calibration curves was based on seven for pulsatilla saponin D, and on eight for ferulic acid concentrations, prepared in triplicate. The detected peak areas were correlated with the corresponding concentrations of reference compounds. The limits of detection (LOD) and limits of quantification (LOQ) under the chromatographic conditions were separately calculated as a signal-to-noise ratio $(\mathrm{S} / \mathrm{N})$ of 3 and 10 , respectively according to The International Council for Harmonisation of Technical Requirements for Pharmaceuticals for Human Use (ICH) guidelines.

Precision and repeatability. The relative standard deviation (RSD) was taken as a measure of precision and repeatability. The intra- and interday variance were measured to obtain the precision of the LC-QTOF-MS method using the standard solutions at two different concentration levels. Each concentration was injected in three replicates on the same day and further on three consecutive days for the determination of the intraday and interday precision, respectively. To investigate the repeatability, five solutions at one concentration were extracted and analyzed.

Recovery. To evaluate the accuracy of the method applied, a recovery test was done by spiking the reference solutions to the $P$. patens sample and measuring the concentrations in triplicate.

Cytotoxicity assays. The assessment of cytotoxicity was made using cell lines obtained from the ATCC (American Type Culture Collection Rockville, MD, USA) in accordance with the methodological procedure used in the National Center for Natural Products Research (University of Mississippi, MS, USA). The cell line panel included five human cancer cell lines-epidermal carcinoma (KB), breast carcinoma (BT-549), ovarian adenocarcinoma (SK-OV-3), skin malignant melanoma (SK-MEL), cervical adenocarcinoma (HeLa) and two noncancerous kidney cell lines - epithelial cells (LLC-PK-1), fibroblast (Vero). Briefly, cells were passaged (25,000 cells/well) into 96-well microplates (tissue culture-treated) and incubated for $24 \mathrm{~h}$. Afterward, different concentrations of the extract were added and incubation continued for $48 \mathrm{~h}$. Finally, after incubation, a modified Neutral Red assay was used to determine the cell viability ${ }^{64}$. The $\mathrm{IC}_{50}$ values were calculated from dose-response curves. The doxorubicin was used as a reference cytotoxic drug (positive control), whereas DMSO was used as a negative control (vehicle control).

Transfection and luciferase assays. HeLa cells (ATCC, Bethesda, MD, USA) were maintained in Dulbecco's modified Eagle's medium (DMEM) (Gibco Life Technologies, Grand Island, NY, USA) containing 10\% fetal bovine serum (FBS, Atlanta Biologicals Inc., Atlanta, GA, USA) and then were plated in white opaque 384 well plates at a density of 4300 cells/well in $30 \mu \mathrm{L}$ of growth medium supplemented with $10 \% \mathrm{FBS}$ and $1 \% \mathrm{Pen} /$ step. The next day, the medium was aspirated and replaced with DMEM containing $10 \%$ FBS only. The cells 
were transfected with respective plasmids using X-tremeGENE HP transfection reagent (Roche Applied Science, Indianapolis, IN, USA). Luciferase vectors used in this assay are summarized in Table 5. Luciferase reporter constructs were transfected into Hela cells as previously described ${ }^{35}$. After $24 \mathrm{~h}$ of transfection, the test agents were added to the transfected cells, followed $30 \mathrm{~min}$ later by an inducing agent: IL-6 (50 ng/mL, R\&D Systems, Inc., Minneapolis, MN, USA) for Stat $3^{35}$, TGF-beta ( $5 \mathrm{ng} / \mathrm{mL}, \mathrm{R} \& D$ Systems, Inc., Minneapolis, MN, USA) for $\mathrm{Smad}^{35}$, Wnt-3a (500 ng/mL, Peprotech Corporation, Rocky Hill, NJ, USA) for Wnt ${ }^{35}$ and PMA (phorbol 12-myristate 13-acetate, 77 ng/mL, Sigma Chemical Company, St. Louis, MO, USA) for AP-1, NF-кB, E2F, MYC, Ets, Notch and Hdghog. No inducer was added for FoxO, miR-21, k-Ras and pTK-control (thymidine kinase promoter). After $4 \mathrm{~h}$ or $6 \mathrm{~h}$ of induction, the cells were lysed by the addition of the One-Glo luciferase assay system (Promega Corporation, Madison, WI, USA). The light output was detected in a Glomax Multi + detection system with Instinct Software (Promega Corporation, Madison, WI, USA). This luciferase assay determines if the test agent was able to inhibit the activation of cancer-related signaling pathways. In the case of FoxO and mi-R21 enhanced luciferase activity by the test agent was assessed ${ }^{17,35}$.

Received: 13 January 2021; Accepted: 30 April 2021

Published online: 20 May 2021

\section{References}

1. Sherris, J., Herdman, C. \& Elias, C. Cervical cancer in the developing world. West. J. Med. 175, 231-233 (2001)

2. Torre, L. A. et al. Global cancer statistics 2012. Cancer J. Clin. 65, 87-108 (2015).

3. Callaway, E. Deal done over HeLa cells line. Nature 500, 132-133 (2013).

4. Adey, A. et al. The haplotype-resolved genome and epigenome of the aneuploid HeLa cancer cell line. Nature 500, 207-211 (2013).

5. Landry, J. et al. The genomic and transcriptomic landscape of a HeLa cell line. G3 Bethesda 8, 1213-1224 (2013).

6. Forment, J. V., Abderrahmane, K. \& Jackson, S. P. Chromothrypsis and cancer: causes and consequences of chromosome shattering. Nat. Rev. Cancer 12, 663-670 (2012).

7. Korbel, J. O. \& Campbell, P. J. Criteria for inference of chromothripsis in cancer genomes. Cell 152, 1226-1236 (2013).

8. Ehrenfeld, E., Modlin, J. \& Chumakov, K. Future of polio vaccines. Expert. Rev. Vaccines 7, 899-905 (2009).

9. Alimonti, J. B., Ball, B. \& Fowke, K. R. Mechanisms of CD4+ T lymphocyte cell heath in human immunodeficiency virus infection and AIDS. J. Gen. Virol. 84, 1649-1661 (2003).

10. Hausen Zur, H. Papillomaviruses and cancer: from basic studies to clinical application. Nat. Rev. Cancer 3, 342-350 (2002).

11. Burk, R. D., Chen, Z. \& Van Doorslaer, K. Human papillomaviruses: genetic basis of carcinogenicity. Public Health Genomics 12, 281-290 (2009).

12. Hausen Zur, H. Papillomaviruses in human cancers. Proc. Assoc. Am. Phys. 111, 581-587 (1999).

13. Wadman, M. Medical research: cell division. Nature 498, 422-426 (2013).

14. Li, W. et al. Oleanane-type triterpenoid saponins from the roots of Pulsatilla koreana and their apoptosis-inducing effects on HL-60 human promyelocytic leukemia cells. Arch. Pharm. Res. 36, 768-774 (2013).

15. Den virtuella floran - Naturhistoriska riksmuseet. http://linnaeus.nrm.se/flora/di (2020).

16. Son, M. K. et al. SB365, Pulsatilla saponin D suppresses proliferation and induces apoptosis of pancreatic cancer cells. Oncol Rep. 30, 801-808 (2013).

17. Hamilton, K. L. et al. Effects of Maackia amurensis seed lectin (MASL) on oral squamous cell carcinoma (OSCC) gene expression and transcriptional signaling pathways. J. Cancer Res. Clin. Oncol. https://doi.org/10.1007/s00432-020-03456-8 (2020).

18. Jin, M. M. et al. UPLC-Q-TOF-MS/MS-guided dereplication of Pulsatilla chinensis to identify triterpenoid saponins. Phytochem. Anal. 29, 516-527 (2018).

19. Zhang, W. et al. A high-throughput metabolomics approach for the comprehensive differentiation of four Pulsatilla Adans herbs combined with a nontargeted bidirectional screen for rapid identification of triterpenoid saponins. Anal. Bioanal. Chem. 411, 2071-2088 (2019).

20. Ye, W. et al. Five new triterpene saponins from Pulsatilla patens var multifida. J. Nat. Prod. 62, 233-237 (1999).

21. Ye, W. C., Zhang, Q. W., Hsiao, W. L. W., Zhao, S. X. \& Che, C. T. Novel cytotoxic lupane-type glycosides from Pulsatilla chinensis. Planta Med. 68, 183-186 (2002).

22. Ling, Y., Lin, Z., Zha, W., Lian, T. \& You, S. Rapid detection and characterisation of triterpene saponins from the root of Pulsatilla chinensis (bunge) regel by HPLC-ESI-QTOF-MS/MS. Phytochem. Anal. 27, 174-183 (2016).

23. Lee, K. Y. et al. Quality control of Pulsatilla koreana based on the simultaneous determination of triterpenoidal saponins by HPLCELSD and principal component analysis. Phytochem. Anal. 21, 314-321 (2010).

24. Shumova, G. S., Savelieva, E. V., Vladymyrova, I. N. \& Tishakova, T. S. Phenolic compound composition of herb of Pulsatilla pratensis (L) Mill. World Sci. 12, 35-38 (2017).

25. Said, R. et al. Tentative characterization of polyphenolic compounds in the male flowers of Phoenix dactylifera by liquid chromatography coupled with mass spectrometry and DFT. Int. J. Mol. Sci. 18, 512 (2017).

26. Bunzel, M., Ralph, J., Brüning, P. \& Steinhart, H. Structural identification of dehydrotriferulic and dehydrotetraferulic acids isolated from insoluble maize bran fiber. J. Agric. Food Chem. 54, 6409-6418 (2006).

27. Mahomoodally, M. F. et al. Utilisation of Rhododendron luteum Sweet bioactive compounds as valuable source of enzymes inhibitors, antioxidant, and anticancer agents. Food Chem. Toxicol. 135, 111052 (2020).

28. Sinan, K. I. et al. Biopotential of Bersama abyssinica fresen stem bark extracts: UHPLC profiles, antioxidant, enzyme inhibitory, and antiproliferative propensities. Antioxidants 9, 163 (2020).

29. Khoza, B. S., Gbashi, S., Steenkamp, P. A., Njobeh, P. B. \& Madala, N. E. Identification of hydroxylcinnamoyl tartaric acid esters in Bidens pilosa by UPLC-tandem mass spectrometry. S. Afr. J. Bot. 103, 95-100 (2016).

30. Kelebek, H., Kadiroğlu, P., Banu Demircan, N. \& Selli, S. Screening of bioactive components in grape and apple vinegars: Antioxidant and antimicrobial potential. J. Inst. Brew. https://doi.org/10.1002/jib.432 (2017).

31. Zengin, G. et al. Antioxidant abilities, key enzyme inhibitory potential and phytochemical profile of Tanacetum poteriifolium Grierson. Ind. Crops Prod. 140, 111629 (2019).

32. Li, Z. H. et al. Rapid identification of flavonoid constituents directly from PTP1B inhibitive extract of raspberry (Rubus idaeus L.) leaves by HPLC-ESI-QTOF-MS-MS. J. Chromatogr. Sci. 54, 805-810 (2016).

33. Laska, G. et al. Phytochemical screening of Pulsatilla species and investigation of their biological activities. Acta Soc. Bot. Pol. 88, 3613. https://doi.org/10.5586/asbp.3613 (2019).

34. Hong, S. W. et al. SB365, Pulsatilla saponin D, targets c-Met and exerts antiangiogenic and antitumor activities. Carcinogenesis 34, 2156-2169 (2013). 
35. Zaki, M. A. et al. Cytotoxicity and modulation of cancer-related signaling by (Z)- and (E)-3,4,3',5'-tetramethoxystilbene isolated from Eugenia brigida. J. Nat. Prod. 76, 679-684 (2013).

36. Tkach, M. et al. p42/p44 MAPK-mediated Stat3Ser727 phosphorylation is required for progestin-induced full activation of Stat3 and breast cancer growth. Endocr-Relat. Cancer 20, 197-212 (2013).

37. Klampfer, L. Signal transducers and activators of transcription (STATs): Novel targets of chemopreventive and chemotherapeutic drugs. Curr. Cancer Drug Targets 6, 107-121 (2006).

38. Massagué, J. TGF-beta in cancer. Cell 134, 215-230 (2008).

39. Cavin, L. G. et al. Transforming growth factoralpha inhibits the intrinsic pathway of c-Myc-induced apoptosis through activation of nuclear factor-kappaB in murine hepatocellular carcinomas. Mol. Cancer Res. 3, 403-412 (2005).

40. Rizzo, P. et al. Rational targeting of Notch signaling in cancer. Oncogene 27, 5124-5131 (2008).

41. Qi, T. et al. Function and regulation of the PEA3 subfamily of ETS transcription factors in cancer. Am. J. Cancer Res. 10, 3083-3105 (2020).

42. Chen, H. Z., Tsai, S. Y. \& Leone, G. Emerging roles of E2Fs in cancer: an exit from cell cycle control. Nat. Rev. Cancer 9, 785-797 (2009).

43. Skoda, A. M. et al. The role of the Hedgehog signaling pathway in cancer: A comprehensive review. Bosn. J. Basic Med. Sci. 18, 8-20 (2018).

44. Tewari, D. et al. Targeting activator protein 1 signaling pathway by bioactive natural agents: possible therapeutic strategy for cancer prevention and intervention. Pharmacol. Res. 128, 366-375 (2018).

45. Roy, S. K., Srivastava, R. K. \& Shankar, S. Inhibition of PI3K/AKT and MAPK/ERK pathways causes activation of FoxO transcription factor, leading to cell cycle arrest and apoptosis in pancreatic cancer. J. Mol. Signal. 5, 10 (2010).

46. Maubant, S. et al. Transcriptome analysis of Wnt3a-treated triple-negative breast cancer cells. PLoS ONE 10, e0122333. https:// doi.org/10.1371/journal.pone.0122333 (2015).

47. Liu, Q. et al. Pulsatilla saponin A, an active molecule from Pulsatilla chinensis, induces cancer cell death and inhibits tumor growth in mouse xenograft models. J. Surg. Res. 188, 387-395 (2014).

48. Kim, Y., Kim, S. B., You, Y. J. \& Ahn, B. Z. Deoxypodophyllotoxin; The cytotoxic and antiangiogenic component from Pulsatilla koreana. Planta Med. 68, 268-271 (2002).

49. Cheon, S. A., Choi, B. K., Jeong, C. S., Li, D. W. \& Lee, E. B. The anti-inflammatory and analgesic actions of the fractions from Pulsatilla koreana root extract. Korean J. Pharm. 31, 174-184 (2000).

50. Laska, G., Sienkiewicz, A., Zjawiony, J. K., Jacob, M. \& Khan, S. Antifungal activity of the root extracts of Pulsatilla patens against Candida glabrata. Acta Pol. Pharm. Drug Res. 74, 179-185 (2017).

51. Chen, Y. Q. et al. Laboratory evaluation of the molluscicidal activity of Pulsatilla chinensis (Bunge) Regel saponins against the snail Oncomelania hupensis. Biomed. Environ. Sci. 25, 224-229 (2012).

52. Anastasiya, V. et al. ROS generation and antioxidant defense systems in normal and malignant cells. Oxid. Med. Cell. Longev. https://doi.org/10.1155/2019/6175804 (2019).

53. Wondrak, G. T. Redox-directed cancer therapeutics: molecular mechanisms and opportunities. Antioxid. Redox Signal. 11, 30133069 (2009).

54. Kim, Y., Bang, S. C., Lee, J. H. \& Ahn, B. Z. Pulsatilla saponin D: the antitumor principle from Pulsatilla koreana. Arch. Pharm. Res. 27, 915-918 (2004).

55. Liu, J. Y. et al. Saponins with neuroprotective effects from the roots of Pulsatilla cernua. Molecules 17, 5520-5531 (2012).

56. Samadder, A., Das, J., Das, S. \& Khuda-Bukhsh, A. R. Dihydroxy-isosteviol-methyl-ester, an active biological component of Pulsatilla nigricans, reduces arsenic induced cellular dysfunction in testis of male mice. Environ. Toxicol. Pharmacol. 34, 743-752 (2012).

57. Sun, H., Wang, Y., Zhang, X. Q., Zhao, S. X. \& Ye, W. C. Chemical constituents of Pulsatilla dahurica. Chem. Nat. Comp. 45, 764-765 (2009).

58. Xu, H. J. et al. A rapid method for simultaneous determination of triterpenoid saponins in Pulsatilla turczaninovii using microwaveassisted extraction and high performance liquid chromatography-tandem mass spectrometry. Food Chem. 135, 251-258 (2012).

59. Xu, Q. M. et al. Antitumor activity of Pulsatilla chinensis (Bunge) Regel saponins in human liver tumor 7402 cells in vitro and in vivo. Phytomedicine 19, 293-300 (2012).

60. Hossain, M. B., Rai, D. K., Brunton, N. P., Martin-Diana, A. B. \& Barry-Ryan, C. Characterization of phenolic composition in Lamiaceae spices by LC-ESI-MS/MS. J. Agric. Food Chem. 58, 10576-10581 (2010).

61. Mazid, M., Khan, T. A. \& Mohammad, F. Role of secondary metabolites in defense mechanisms of plants. Biol. Med. 3, 232-249 (2011).

62. Carazzone, C., Mascherpa, D., Gazzani, G. \& Papetti, A. Identification of phenolic constituents in red chicory salads (Cichorium intybus) by high-performance liquid chromatography with diode array detection and electrospray ionisation tandem mass spectrometry. Food Chem. 138, 1062-1071 (2013).

63. Xu, H. et al. Qualitative and quantitative determination of nine main active constituents in Pulsatilla cernua by high-performance liquid chromatography coupled to electrospray ionization tandem mass spectrometry. J. Sep. Sci. 34, 308-316 (2011).

64. Borenfreund, E., Babich, H. \& Martin-Alguacil, N. Rapid chemosensitivity assay with human normal and tumor cells in vitro. Vitro Cell. Dev. Biol. 26, 1030-1034 (1990).

\section{Acknowledgements}

This study was supported by a grant nr WZ/WB-IIS/2/2020 from the Ministry of Science and Higher Education of Poland to GŁ and in part by a contract from the University of Mississippi Medical Center Cancer Institute Agreement \#64580110000 to PB and DP.

\section{Author contributions}

G.Ł. conceptualization, G.Ł., E.S., M.MT., Ł.Ś., P.B., D.P. methodology and investigation, G. Ł., E.S., M.MT., Ł.Ś. contributed to writing/original draft preparation and editing, G. $€$. funding. All authors reviewed the manuscript.

\section{Competing interest}

The authors declare no competing interests.

Additional information

Supplementary Information The online version contains supplementary material available at https://doi.org/ 10.1038/s41598-021-90136-3.

Correspondence and requests for materials should be addressed to E.S. 
Reprints and permissions information is available at www.nature.com/reprints.

Publisher's note Springer Nature remains neutral with regard to jurisdictional claims in published maps and institutional affiliations.

(c) (i) Open Access This article is licensed under a Creative Commons Attribution 4.0 International License, which permits use, sharing, adaptation, distribution and reproduction in any medium or format, as long as you give appropriate credit to the original author(s) and the source, provide a link to the Creative Commons licence, and indicate if changes were made. The images or other third party material in this article are included in the article's Creative Commons licence, unless indicated otherwise in a credit line to the material. If material is not included in the article's Creative Commons licence and your intended use is not permitted by statutory regulation or exceeds the permitted use, you will need to obtain permission directly from the copyright holder. To view a copy of this licence, visit http://creativecommons.org/licenses/by/4.0/.

(C) The Author(s) 2021 